\title{
Elliptic genera of non-compact Gepner models and mirror symmetry
}

\author{
Sujay K. Ashok ${ }^{a}$ and Jan Troost ${ }^{b}$ \\ ${ }^{a}$ Institute of Mathematical Sciences, C.I.T Campus, Taramani, \\ Chennai, 600113 India \\ ${ }^{b}$ Laboratoire de Physique Théorique, ${ }^{1}$ Ecole Normale Supérieure, \\ 24 rue Lhomond, F-75231 Paris Cedex 05, France \\ E-mail: sashok@imsc.res.in, troost@lpt.ens.fr
}

ABSTRACT: We consider tensor products of $N=2$ minimal models and non-compact conformal field theories with $N=2$ superconformal symmetry, and their orbifolds. The elliptic genera of these models give rise to a large and interesting class of real Jacobi forms. The tensor product of conformal field theories leads to a natural product on the space of completed mock modular forms. We exhibit families of non-compact mirror pairs of orbifold models with $c=9$ and show explicitly the equality of elliptic genera, including contributions from the long multiplet sector. The Liouville and cigar deformed elliptic genera transform into each other under the mirror transformation.

Keywords: Conformal and W Symmetry, Extended Supersymmetry, Conformal Field Models in String Theory

ARXIV EPRINT: 1204.3802

\footnotetext{
${ }^{1}$ Unité Mixte du CNRS et de l'Ecole Normale Supérieure associée à l'université Pierre et Marie Curie 6, UMR 8549.
} 


\section{Contents}

1 Introduction $\quad 2$

2 Elliptic genera 3

2.1 Definition and properties 3

2.2 The building blocks 3

2.2.1 The $N=2$ minimal models 4

2.2.2 The $N=2$ Liouville model 4

2.2.3 The coset conformal field theory 5

2.3 Tensor product theories 5

2.4 Twisted blocks 6

3 Mirror symmetry for Gepner models $\quad 7$

$\begin{array}{lll}3.1 & \text { Non-compact Gepner models } & 7\end{array}$

$\begin{array}{lll}3.2 & \text { Mirror symmetry through orbifolds } & 8\end{array}$

3.3 Models with central charge $c=6 \quad 8$

3.3.1 The ground states 9

$\begin{array}{lll}3.3 .2 & \text { Mirror symmetry }\end{array}$

4 Models with central charge $c=9 \quad 10$

4.1 The $(2 k, 2 k ; k)$ model 11

$\begin{array}{lll}\text { 4.1.1 An infinite family of mirror pairs } & 12\end{array}$

$\begin{array}{lll}4.1 .2 & \text { The long multiplet sector } & 14\end{array}$

$\begin{array}{ll}4.2 \text { The }(k ; 2 k, 2 k) \text { model } & 16\end{array}$

$\begin{array}{lll}\text { 4.2.1 The short multiplet bound states } & 16\end{array}$

$\begin{array}{lll}\text { 4.2.2 The long multiplet scattering states } & 17\end{array}$

5 Notes on mock modular forms $\quad 19$

$\begin{array}{ll}5.1 \text { The shadow } & 19\end{array}$

$\begin{array}{ll}5.2 & \text { The product of mock modular forms } \\ 5.3 & 20\end{array}$

5.3 The orbifolds of completions of mock modular forms 20

$\begin{array}{ll}5.4 \text { Uniqueness } & 21\end{array}$

A Characters $\quad 22$

A.1 Minimal model characters 22

$\begin{array}{ll}\text { A.2 Minimal model twisted blocks } & 22\end{array}$

A.3 The $\mathbb{Z}_{k}$ orbifold and mirror symmetry 23

$\begin{array}{lll}\text { A.4 Characters at } c>3 & 24\end{array}$

A.5 Twisted building blocks at $c>3 \quad 25$

$\begin{array}{lll}\text { A.5.1 Character formulae } & 25\end{array}$

A.5.2 Twisted blocks for the non-holomorphic sector 25

A.5.3 Exact expressions for twisted blocks 26 


\section{Introduction}

The study of two-dimensional conformal field theories in terms of their minimal model description, their Landau-Ginzburg phase or as gauged linear sigma-models has proven to be very useful $[1,2]$. It has taught us about the space of two-dimensional conformal field theories, and its geometrical structure. The study has had a profound impact on our understanding of compact Calabi-Yau manifolds and mirror symmetry, and it has had interesting applications in the field of singular manifolds and toric varieties (see [3] for a review).

The extension of this study to include theories with non-compact targets, and in particular non-compact Calabi-Yau manifolds is very interesting. It is a natural generalization from the perspective of studying Calabi-Yau manifolds locally, or from the viewpoint of understanding holography in curved non-compact spaces that asymptotically have a linear dilaton profile $[4,5]$. This field has already given rise to many results including a study of the map between deformations of the geometry and the spectrum of non-compact conformal field theories [6-10], mirror symmetry for non-compact Gepner models, as well as an intriguing relation between orbifolds in asymptotically linear dilaton spaces and flat space toric orbifolds [10]. However many of the results have been based on studying the chiral (anti-chiral) rings of the theory.

Recently, there has been a lot of progress in our understanding of the elliptic genus of non-compact $N=2$ superconformal field theories with central charge larger than three [1113]. In particular, it was understood that the elliptic genus is modular covariant and real. Non-holomorphic contributions arise from the continuous part of the spectrum of the twodimensional conformal field theory. This has led to a physical understanding of the modular completion of mock modular forms in terms of both a modular Lagrangian path integral description [11], and a Hamiltonian viewpoint in terms of an integral over a difference of spectral densities for right-moving primary bosons and fermions [13].

In this paper we apply these new insights to the study of conformal field theories which are tensor products of $N=2$ minimal models and $N=2$ Liouville theories (or $N=2$ cigar coset models), and their orbifolds. The elliptic genus of tensor product theories is the product of the individual elliptic genera. For orbifold theories, we can often identify the elliptic genus via standard twisting procedures.

For orbifolds of products of compact Gepner models, there have been many interesting results [14-18], especially in the context of mirror symmetry. Given a Landau-Ginzburg formulation of a compact Calabi-Yau, there is an algorithmic way to construct the mirror. Most of the results on elliptic genera rely on the fact that, given the Poincaré polynomial of the target space, there is a unique extension to the elliptic genus and the identification of mirror pairs thus becomes simpler.

For non-compact conformal field theories, our generic construction will give rise to a large new class of real Jacobi forms. In particular, when more than one non-compact model is involved, the product of elliptic genera gives rise to a modular completion of the product of mock modular forms. Conformal field theory elliptic genera thus provide a natural way to complete the product of two mock modular forms.

We apply this general reasoning to non-compact Gepner models and their orbifolds in 
type II string theory. The knowledge we gained about non-compact elliptic genera allows us to check mirror symmetry explicitly in these models in a way coherent with modularity and ellipticity. The check includes some long multiplet contributions. Our analysis is constructive in the sense that, starting from the elliptic genus of a given orbifold theory we rewrite it such that the final expression has a natural interpretation as the elliptic genus of the mirror model. Under the mirror transform, the Liouville factors naturally go over into their cigar counterparts.

The paper has the following organization. In section 2 we discuss the elliptic genera of the basic models, which are the minimal models with $c<3$ and the two types of noncompact models with $c>3$, the Liouville and cigar theories. We also describe how to put these together and construct the elliptic genera of tensor product and orbifold models. In section 3 we restrict to orbifold models which are non-compact generalizations of Gepner models in type II string theory. Examples with central charge $c=6$ are provided in section 3.3 and those with central charge $c=9$ are discussed in section 4 . The technical ingredients necessary for the calculations in these sections are provided in appendix A. We end in section 5 with a number of proposals for how to extend our class of examples to a broader domain.

\section{Elliptic genera}

In this section, we review the elliptic genera of $N=2$ minimal models and $N=2$ superconformal field theories with central charge greater than three, since these conformal field theories form the building blocks of the models we study in sections 3 and 4 . We also pause to make a point about an embryonic example of mirror symmetry.

\subsection{Definition and properties}

We study $N=2$ superconformal field theories with a left $\mathrm{U}(1)_{R}$ charge $J_{0}$ and a right charge $\bar{J}_{0}$, as well as scaling dimension operators $L_{0}$ and $\bar{L}_{0}$. The elliptic genus $\chi[19,20]$ is defined as a twisted partition sum with periodic boundary conditions for the fermions:

$$
\chi(q, z)=\operatorname{Tr}(-1)^{F} q^{L_{0}-\frac{c}{24}} \bar{q}^{\tilde{L}_{0}-\frac{c}{24}} z^{J_{0}}
$$

We will also use the notation $\chi(q, z) \equiv \chi(\tau, \alpha)$ for the elliptic genus, where the arguments are related through the equations $q=e^{2 \pi i \tau}$ and $z=e^{2 \pi i \alpha}$. The elliptic genus has elliptic and modular covariance properties which make it a Jacobi form.

\subsection{The building blocks}

In this subsection, we list the elliptic genera of the elementary building blocks that we will use to construct our models. 


\subsubsection{The $N=2$ minimal models}

The elliptic genus of an $N=2$ minimal model with central charge $c=3-\frac{6}{k}$ and $k$ a positive integer is given by [1]:

$$
\chi(k ;-)(q, z)=\frac{\theta_{11}\left(q, z^{\frac{k-1}{k}}\right)}{\theta_{11}\left(q, z^{\frac{1}{k}}\right)} .
$$

This is also the elliptic genus of the compact Landau-Ginzburg model with superpotential $W=X^{k}$. It can, moreover, be derived from the gauged Wess-Zumino-Witten description of the model. We denote the level of the minimal model as an extra argument for the elliptic genus, followed by a semicolon. The elliptic genus of the minimal model has an expansion in terms of twisted Ramond sector characters [1, 21]:

$$
\chi(k ;-)(q, z)=\sum_{j=0, \frac{1}{2}, \ldots}^{\frac{k-2}{2}} \mathcal{C}_{2 j+1}^{j}(q, z) .
$$

The basic definitions and the modular and elliptic properties of these characters are reviewed in appendix $\mathrm{A}$.

\subsubsection{The $N=2$ Liouville model}

Next, we consider models with central charge $c=3+\frac{6}{l}$ with the level $l$ equal to a positive integer. The $\mathbb{Z}_{l} \subset \mathrm{U}(1)_{R}$ orbifold of the $\mathrm{SL}(2, \mathbb{R})_{l} / \mathrm{U}(1)$ coset theory with central charge $c=3+\frac{6}{l}$ has elliptic genus [11]:

$$
\chi(-; l)(q, z)=\frac{i \theta_{11}(q, z)}{\eta^{3}} \hat{A}_{2 l}\left(z^{\frac{1}{l}}, z^{2} ; q\right)
$$

The level $l$ of the non-compact model follows the semicolon. This elliptic genus is also the genus of a generalized non-compact Landau-Ginzburg model with superpotential $W=$ $e^{-l Y}$, coinciding with $N=2$ Liouville theory at radius $R=\sqrt{l \alpha^{\prime}}$.

Let us discuss these points in some detail, since it provides an important embryonic example of mirror symmetry that pervades the rest of our paper. Note that there are two known ways to obtain the expression (2.4) for the elliptic genus. The first way is through the non-compact Landau-Ginzburg model, where one identifies the R-charges of the fields, and their proper configuration space, then to do a free field calculation to obtain the holomorphic part of the elliptic genus [11]. A scattering calculation using the LandauGinzburg potential will then further provide the remainder term in the elliptic genus [13], thus proving that expression (2.4) is the elliptic genus of $N=2$ Liouville theory at radius $R=\sqrt{l \alpha^{\prime}}$. Alternatively, a path integral calculation shows that this is also the elliptic genus of the $\mathbb{Z}_{l}$ orbifold of the cigar coset conformal field theory [11, 13]. This provides further evidence for the equivalence of these models $[22,23]$ in terms of the match of a modular covariant partition sum. 
The mock modular form, of which the elliptic genus is the completion, is a holomorphic Appell-Lerch sum which has an expansion in terms of twisted Ramond $N=2$ superconformal characters $C h$ extended by spectral flow (see [23, 24] for our conventions for the arguments):

$$
\chi_{h o l}(-; l)(q, z)=\sum_{2 j-1=0}^{l-1} C h\left(j ;-\frac{1}{2} ; q, z\right) .
$$

\subsubsection{The coset conformal field theory}

The $\mathrm{SL}(2, \mathbb{R})_{l} / \mathrm{U}(1)$ supersymmetric coset theory, which we refer to as the cigar theory, has an elliptic genus obtained by taking the $\mathbb{Z}_{l}$ orbifold of the elliptic genus quoted above. It is given by

$$
\chi(-; l)^{\mathbb{Z}_{l}}(q, z)=\frac{1}{l} \frac{i \theta_{11}(q, z)}{\eta^{3}} \sum_{m_{a}, m_{b} \in \mathbb{Z}_{l}} e^{-\frac{2 \pi i m_{a} m_{b}}{l}} q^{-\frac{m_{a}^{2}}{l}} \hat{A}_{2 l}\left(z^{\frac{1}{l}} q^{\frac{m_{b}}{l}} e^{\frac{2 \pi i m_{b}}{l}}, z^{2} ; q\right) .
$$

We have denoted the orbifold group as a superscript to the elliptic genus. It is also the elliptic genus of $N=2$ Liouville theory at radius $R=\sqrt{\alpha^{\prime} / l}$. The holomorphic part of the elliptic genus can again be expanded in terms of the extended characters:

$$
\chi_{h o l}(-; l)^{\mathbb{Z}_{l}}(q, z)=\sum_{2 j-1=0}^{l-1} C h\left(j ;-\frac{1}{2}-(2 j-1) ; q, z\right) .
$$

\subsection{Tensor product theories}

An elementary but important point is that the elliptic genus of a tensor product conformal field theory is the product of the individual elliptic genera:

$$
\chi\left(\otimes_{i} C F T_{i}\right)=\prod_{i} \chi\left(C F T_{i}\right) .
$$

For example, for the tensor product of compact and non-compact Landau-Ginzburg models with central charges associated to the positive and integer levels $\left(k_{1}, k_{2}, \ldots, k_{p} ; l_{1}, l_{2}, \ldots, l_{q}\right)$, the elliptic genus reads:

$$
\chi\left(k_{1}, k_{2}, \ldots, k_{p} ; l_{1}, l_{2}, \ldots, l_{q}\right)(q, z)=\prod_{i=1}^{p} \frac{\theta_{11}\left(q, z^{1-\frac{1}{k_{i}}}\right)}{\theta_{11}\left(q, z^{\frac{1}{k_{i}}}\right)} \prod_{j=1}^{q} \frac{i \theta_{11}(q, z)}{\eta^{3}} \hat{A}_{2 l_{j}}\left(z^{\frac{1}{l_{j}}}, z^{2} ; q\right) .
$$

One can generalize this elliptic genus to one which keeps track of the R-charges of the individual factor theories. We find a generalized elliptic genus:

$$
\chi\left(k_{1}, k_{2}, \ldots, k_{p} ; l_{1}, l_{2}, \ldots, l_{q}\right)\left(q, z_{i}, z_{j}\right)=\prod_{i=1}^{p} \frac{\theta_{11}\left(q, z_{i}^{1-\frac{1}{k_{i}}}\right)}{\theta_{11}\left(q, z_{i}^{\frac{1}{k_{i}}}\right)} \prod_{j=1}^{q} \frac{i \theta_{11}\left(q, z_{j}\right)}{\eta^{3}} \hat{A}_{2 l_{j}}\left(z_{j}^{\frac{1}{l_{j}}}, z_{j}^{2} ; q\right) .
$$

This is one of many generalizations of the twisted index. One can write down similar expressions where we replace some of the Liouville factors with cigar coset theories. 


\subsection{Twisted blocks}

In the following, we consider orbifolds of tensor products of the above models. For simplicity, we restrict our orbifold groups to be discrete subgroups of the product of the $\mathrm{U}(1)$ R-symmetry groups of the factor models. In these circumstances, it is straightforward to generalize the techniques of [14] to describe the twisted partition sums from which we build the elliptic genus of the orbifold. In each factor theory, we have partition sums in the sectors twisted by the generator of an orbifold group $\mathbb{Z}_{n}$ to the power $m_{a} \in \mathbb{Z}_{n}$ and we can insert an operator corresponding to a generator of the orbifold group to the power $m_{b} \in \mathbb{Z}_{n}$. We then obtain the twisted partition functions:

$$
\chi_{m_{a}, m_{b}}(q, z)=e^{2 \pi i \frac{c}{6} m_{a} m_{b}} e^{2 \pi i \frac{c}{6}\left(m_{a}^{2} \tau+2 m_{a} \alpha\right)} \chi\left(\tau, \alpha+m_{a} \tau+m_{b}\right) .
$$

The transformation properties of these twisted elliptic genera are (with $\lambda, \mu \in \mathbb{Z}$ ):

$$
\begin{aligned}
\chi_{m_{a}, m_{b}}\left(-\frac{1}{\tau}, \frac{\alpha}{\tau}\right) & =e^{2 \pi i \frac{c}{6} \frac{\alpha^{2}}{\tau}} \chi_{m_{b},-m_{a}}(\tau, \alpha) \\
\chi_{m_{a}, m_{b}}(\tau+1, \alpha) & =\chi_{m_{a}+m_{b}, m_{b}}(\tau, \alpha) \\
\chi_{m_{a}, m_{b}}(\tau, \alpha+\lambda \tau+\mu) & =e^{2 \pi i \frac{c}{6}\left(m_{a} \mu-m_{b} \lambda-\lambda \mu\right)} e^{-2 \pi i \frac{c}{6}\left(\lambda^{2} \tau+2 \lambda \alpha\right)} \chi_{m_{a}+\lambda, m_{b}+\mu}(\tau, z) .
\end{aligned}
$$

We assign a canonical phase factor to each factor model:

$$
\epsilon\left(m_{a}, m_{b}\right)=(-1)^{m_{a}+m_{b}+m_{a} m_{b}}
$$

which will ensure that the total orbifolded model is free of discrete torsion. For the partition sum including the phase, we use the notation:

$$
\tilde{\chi}_{m_{a}, m_{b}}=\epsilon\left(m_{a}, m_{b}\right) \chi_{m_{a}, m_{b}} .
$$

The twisted building blocks for the R-symmetry orbifolds can be simplified using the ellipticity and modular properties of theta functions and completed Appell-Lerch sums $\hat{A}$ (see appendix A for details). It will be convenient to express the twisted building blocks $\tilde{\chi}_{m_{a}, m_{b}}$ of the minimal models and the non-compact conformal field theories in terms of the twisted Ramond sector characters of the conformal field theory. This renders the transformation properties of each term under the insertion of a generator in the trace manifest. We find the twisted blocks:

- for the minimal models

$$
\tilde{\chi}_{m_{a}, m_{b}}(k ;-)=e^{-\frac{2 \pi i m_{a} m_{b}}{k}} \sum_{j=0, \frac{1}{2}, \ldots}^{\frac{k-2}{2}} e^{\frac{2 \pi i m_{b}}{k}(2 j+1)} \mathcal{C}_{2 j+1-2 m_{a}}^{j}(q, z) .
$$

- for the anti-diagonal (or $\mathbb{Z}_{k}$ orbifolded) minimal models

$$
\tilde{\chi}_{m_{a}, m_{b}}(k ;-)^{\mathbb{Z}_{k}}=e^{-\frac{2 \pi i m_{a} m_{b}}{k}} \sum_{j=0, \frac{1}{2}, \ldots}^{\frac{k-2}{2}} e^{-\frac{2 \pi i m_{b}}{k}(2 j+1)} \mathcal{C}_{-2 j-1-2 m_{a}}^{j}(q, z) .
$$


- for the holomorphic part of a Liouville factor:

$$
\tilde{\chi}_{h o l ; m_{a}, m_{b}}(-; l)=e^{\frac{2 \pi i m_{a} m_{b}}{l}} \sum_{2 j-1=0}^{l-1} e^{\frac{2 \pi i m_{b}}{l}(2 j-1)} C h\left(j ;-\frac{1}{2}+m_{a} ; q, z\right) .
$$

- for the holomorphic part of a cigar factor:

$$
\tilde{\chi}_{h o l ; m_{a} . m_{b}}(-; l)^{\mathbb{Z}_{l}}=e^{\frac{2 \pi i m_{a} m_{b}}{l}} \sum_{2 j-1=0}^{l-1} e^{-\frac{2 \pi i m_{b}}{l}(2 j-1)} C h\left(j ;-\frac{1}{2}-(2 j-1)+m_{a} ; q, z\right) \text {. }
$$

The completed twisted blocks for non-compact factors are recorded in appendix A.

\section{Mirror symmetry for Gepner models}

In this section we recapitulate the construction of mirror Gepner models [26], generalized to include non-compact conformal field theories.

\subsection{Non-compact Gepner models}

Gepner's construction of string compactifications in terms of exactly solvable $N=2$ superconformal field theories [25] can be suitably extended to include factor models with central charge larger than 3 (see e.g. [10]). We study non-compact Gepner models consisting of $p$ minimal models at levels $k_{i}$ and $q$ non-compact models at levels $l_{j}$ tensored with $\mathbb{R}^{d-1,1}$. They can be characterized in the light-cone as having a

$$
\mathrm{U}(1)_{2}^{\frac{d-2}{2}} \times \mathrm{U}(1)_{2}^{p+q} \times \prod_{i=1}^{p} \mathrm{U}(1)_{k_{i}} \times \prod_{j=1}^{q} \mathrm{U}(1)_{l_{j}}
$$

worldsheet current algebra. The level 2 factors refer to worldsheet fermion numbers, and the U(1) current algebras at level $k_{i}$ and $l_{j}$ are the R-currents of compact and non-compact $N=2$ superconformal field theories. We have the corresponding charge vectors $r$ :

$$
r=\left(s_{-\frac{d-4}{2}}, \ldots, s_{0}, s_{1}, \ldots, s_{p+q} ; n_{1}, \ldots, n_{p} ;-2 m_{1}, \ldots,-2 m_{q}\right),
$$

with inner product:

$$
r^{(1)} \cdot r^{(2)}=-\frac{s_{-\frac{d-4}{2}}^{(1)} s_{-\frac{d-4}{2}}^{(2)}}{4} \cdots+\frac{n_{1}^{(1)} n_{2}^{(2)}}{2 k_{1}} \cdots-\frac{2 m_{1}^{(1)} 2 m_{2}^{(2)}}{2 l_{1}} \ldots
$$

We introduce a vector $\beta_{0}$ such that twice its inner product with the left-moving charge vector is proportional to the left-moving $\mathrm{R}$-charge. It satisfies $\beta_{0} \cdot \beta_{0}=-1$. We fix conventions such that $\beta_{0}$ is equal to:

$$
\beta_{0}=(1, \ldots, 1,1, \ldots, 1 ; 1, \ldots, 1 ; 1, \ldots, 1) .
$$

If we start from a model diagonal in the charge lattice quantum numbers, then we must perform an orbifold to render the model local on the worldsheet, in the sense of containing only purely NS or purely Ramond states. The necessary $\mathbb{Z}_{2}^{p+q+(d-4) / 2}$ orbifold involves discrete torsion [26]. To obtain the type II Gepner model, one further performs an integer R-charge orbifold, and a $\mathbb{Z}_{2}$ GSO projection. 


\subsection{Mirror symmetry through orbifolds}

Mirror symmetry is implemented in Gepner models through orbifolding by a subgroup of the discrete group $G_{\text {phase }}=\prod_{i=1}^{p} \mathbb{Z}_{k_{i}} \times \prod_{j=1}^{q} \mathbb{Z}_{l_{j}}$ of the $\mathrm{U}(1)_{R}$ symmetries of the factor theories [26]. The integer R-charge orbifold $\mathbb{Z}_{n}$ is already such a subgroup. ${ }^{1}$ The maximal subgroup $H$ of the group $G_{\text {phase }}$ which preserves space-time supersymmetry gives rise to the mirror theory. Thus, the group $H$ will be the maximal subgroup of $G_{\text {phase }} / \mathbb{Z}_{n}$ which preserves the condition that the left and right $\mathrm{R}$-charge remain integer. Let us denote the original Gepner model by $M_{1}$, and the mirror model by $M_{2}=M_{1} / H$. Then if we consider orbifolds of theory $M_{1}$ by the subgroup $H_{1} \subset H$, we will find that the theory $M_{1} / H_{1}$ is mirror to the theory $M_{2} /\left(H / H_{1}\right)$.

We further note that the maximal allowed orbifold will give rise to (the GSO projection of) the T-dual of the original model (before GSO). In the T-dual, all left-moving angular momenta will have an opposite sign. These statements are true for the compact theory because a $\mathbb{Z}_{k}$ orbifold of the minimal model gives rise to its T-dual. For a singular noncompact theory (described by a purely linear dilaton background), the statement also holds. For the deformed or resolved non-compact theories, we need a mild modification. For instance, the $\mathbb{Z}_{l}$ orbifold of Liouville theory at radius $R=\sqrt{l \alpha^{\prime}}$ is Liouville theory at radius $R=\sqrt{\alpha^{\prime} / l}$. That is T-dual to the cigar theory at radius $R=\sqrt{l \alpha^{\prime}}$. Thus orbifolding is equivalent to T-duality only for the compact factors. For the non-compact factors, we must combine orbifolding with an exchange of deformation and resolution in order to obtain the T-dual model. We confirm this picture by the direct evaluation of elliptic genera for the mirror pair.

\subsection{Models with central charge $c=6$}

In this subsection, we get our feet wet with simple examples of non-compact Gepner models with central charge $c=6$, and make some preliminary observations. We concentrate on models involving one compact and one non-compact model at equal levels. As a starting point, we take a product of a minimal model with central charge $c=3-6 / k$ and an $N=2$ Liouville theory at radius $R=\sqrt{k \alpha^{\prime}}$ with central charge $c=3+6 / k$. The integer $\mathrm{R}$ charge orbifold is an orbifold by the group $\mathbb{Z}_{k}$. The conformal field theory describes strings propagating on a space which is asymptotically locally flat, with a linear dilaton slope. It has a deformed $\mathbb{C}^{2} / \mathbb{Z}_{k}$ singularity at the center. Asymptotically, it is T-dual to $S^{3} \times \mathbb{R}$ with a linear dilaton along the non-compact radial direction, and $k$ units of three-form $H_{(3)}$ flux on the three-sphere. Near the center, the geometry is deformed to the T-dual of $k$ NS5-branes spread on a circle in a two-dimensional plane, in a doubly scaled near-horizon limit. See $[10,27]$ for detailed discussions. The elliptic genus of this theory is given by the

\footnotetext{
${ }^{1}$ Here we ignore the fermionic entries in the charge vectors, which we can do if we allow for flat space charge conjugation.
} 
orbifold formula applied to the two factor theories:

$$
\begin{aligned}
& \chi(k ; k)^{\mathbb{Z}_{k}}= \frac{1}{k} \sum_{m_{a}, m_{b}=0}^{k-1} \tilde{\chi}_{m_{a}, m_{b}}(k ;-) \tilde{\chi}_{m_{a}, m_{b}}(-; k) \\
&= \frac{1}{k} \frac{i \theta_{11}(q, z)}{\eta(q)^{3}} \sum_{m_{a}, m_{b}=0}^{k-1} \sum_{j=0, \frac{1}{2}, \ldots}^{\frac{k-2}{2}} e^{\frac{2 \pi i m_{b}(2 j+1)}{k}} q^{\frac{m_{a}^{2}}{k}} z^{\frac{2 m_{a}}{k}} \\
& \mathcal{C}_{2 j+1-2 m_{a}}^{j}(q, z) \hat{A}_{2 k}\left(z^{\frac{1}{k}} q^{\frac{m_{a}}{k}} e^{\frac{2 \pi i m_{b}}{k}}, z^{2} q^{2 m_{a}} ; q\right) .
\end{aligned}
$$

\subsubsection{The ground states}

To link our results to known results on massless states, we observe that we can recuperate the Poincaré polynomial of the target space from these expressions, by taking the limit that projects onto left-moving (R-charged weighted) Ramond ground states. One thus recovers the results described for instance in [10].

\subsubsection{Mirror symmetry}

To advance our analysis of mirror symmetry we first observe that, for this $c=6$ model, the maximal group $H$ of phase symmetries that we can mod out by while preserving supersymmetry is trivial. Thus, the model must be self-mirror. This can also be seen as a consequence of the (generalized) hyperkähler structure of the target space. We conclude that the elliptic genus of the model has to be equal to the elliptic genus of a diagonal minimal model times the cigar model at radius $\sqrt{k \alpha^{\prime}}$ modded out by the integer $\mathrm{R}$-charge and GSO projection. The latter elliptic genus is given by:

$$
\begin{aligned}
& \chi^{\prime}(k ; k)^{\mathbb{Z}_{k}=}=\frac{1}{k} \sum_{m_{a}, m_{b}=0}^{k-1} \tilde{\chi}_{m_{a}, m_{b}}(k ;-) \tilde{\chi}_{m_{a}, m_{b}}(-; k)^{\mathbb{Z}_{k}} \\
&=\frac{1}{k^{2}} \frac{i \theta_{11}(\tau, \alpha)}{\eta^{3}} \sum_{m_{a}, m_{b}=0}^{k-1} \sum_{j=0, \frac{1}{2}, \ldots}^{\frac{k-1}{2}} e^{\frac{2 \pi i m_{b}(2 j+1)}{k}} q^{\frac{m_{a}^{2}}{k}} z^{\frac{2 m_{a}}{k}} \mathcal{C}_{2 j+1-2 m_{a}}^{j}(q, z) \\
& \times \sum_{m_{a}^{\prime}, m_{b}^{\prime} \in \mathbb{Z}_{k}} q^{-\frac{m_{a}^{\prime 2}}{k}} e^{-\frac{2 \pi i m_{a}^{\prime} n_{a}^{\prime}}{k}} \hat{A}_{2 l}\left(z^{\frac{1}{k}} q^{\frac{m_{a}+m_{a}^{\prime}}{k}} e^{\frac{2 \pi i\left(m_{b}+m_{b}^{\prime}\right)}{k}}, z^{2} q^{2 m_{a}} ; q\right) .
\end{aligned}
$$

The equality of the elliptic genera in equations (3.5) and (3.6) is non-trivial. To prove the equality, it is useful to render the $N=2$ superconformal representation content of the compact and non-compact elliptic genera manifest. In particular, let us write the holomorphic part of the elliptic genus (3.5) in terms of the characters of the minimal model and the analogous extended characters (2.15) and (2.17):

$$
\chi_{h o l}(k ; k)^{\mathbb{Z}_{k}}=\sum_{j_{1}, j_{2}} \sum_{m_{a}, m_{b} \in \mathbb{Z}_{k}} e^{\frac{2 \pi i m_{b}}{k}\left[\left(2 j_{1}+1\right)+\left(2 j_{2}-1\right)\right]} \mathcal{C}_{2 j_{1}+1-2 m_{a}}^{j_{1}}(q, z) C h\left(j_{2} ;-\frac{1}{2}+m_{a} ; q, z\right) .
$$


The sum over $m_{b}$ imposes the GSO constraint and relates the spin of the two individual factors. In order to render the mirror interpretation manifest, we shift the twisted sector label $m_{a}$ by $-\left(2 j_{2}-1\right)$. We then use the integer R-charge constraint in the angular momentum quantum number of the minimal model character, to end up with the final expression:

$$
\begin{aligned}
\chi_{h o l}(k ; k)^{\mathbb{Z}_{k}}=\sum_{j_{1}, j_{2}} \sum_{m_{a}, m_{b} \in \mathbb{Z}_{k}} e^{\frac{2 \pi i m_{b}}{k}\left[\left(2 j_{1}+1\right)+\left(2 j_{2}-1\right)\right]} \\
\mathcal{C}_{-2 j_{1}-1-2 m_{a}}^{j_{1}}(q, z) \operatorname{Ch}\left(j_{2} ;-\frac{1}{2}+m_{a}-\left(2 j_{2}-1\right) ; q, z\right)(3.8
\end{aligned}
$$

Repackaging this in terms of the twisted blocks, we find

$$
\chi(k ; k)^{\mathbb{Z}_{k}}=\frac{1}{k} \sum_{m_{a}, m_{b}=0}^{k-1} \tilde{\chi}_{m_{a}, m_{b}}(k ;-)^{\mathbb{Z}_{k}} \tilde{\chi}_{h o l ; m_{a}, m_{b}}(-; k)^{\mathbb{Z}_{k}}
$$

We recognize this to be the elliptic genus of an anti-diagonal minimal model times the cigar theory at $R=\sqrt{k \alpha^{\prime}}$, the whole orbifolded by $\mathbb{Z}_{k}$. Performing a similar calculation for the non-holomorphic long multiplet contributions gives rise to the modular completion of the above formula.

In order to fully appreciate the relation between expressions (3.5) and (3.6), we have to go a bit further. We will give more details in the intricate $c=9$ examples, but we already outline the idea here. We wish to re-interpret the mirror model as an orbifold of a diagonal model. For this purpose, we note that the elliptic genus of the anti-diagonal minimal model is related to the diagonal minimal model elliptic genus through a sign flip in the second argument $\alpha$, and the addition of an overall sign (see equation (A.6)). Analogously the noncompact elliptic genus is invariant under such a sign flip (see equation (A.21)). Rewriting in terms of the twisted blocks we find

$$
\begin{aligned}
\chi(k ; k)^{\mathbb{Z}_{k}}(\tau, \alpha) & =-\frac{1}{k} \sum_{m_{a}, m_{b}=0}^{k-1} \tilde{\chi}_{m_{a}, m_{b}}(k ;-)(\tau,-\alpha) \tilde{\chi}_{m_{a}, m_{b}}(-; k)^{\mathbb{Z}_{k}}(\tau, \alpha) \\
& =-\chi^{\prime}(k ; k)^{\mathbb{Z}_{k}}(\tau,-\alpha) .
\end{aligned}
$$

Therefore the elliptic genus of the original model is self-mirror and furthermore equal to the elliptic genus (3.6), up to an overall sign and a sign flip in the second argument $\alpha$. The calculation provides a proof of a non-trivial relation between products and sums of thetafunctions and completed Appell-Lerch sums. In the following sections, we will consider more involved examples of mirror symmetry, including infinite families of mirror pairs, and many more details on the long multiplet contributions, in the context of non-compact Gepner models with central charge $c=9$.

\section{Models with central charge $c=9$}

In this section we will study two types of models with central charge $c=9$. The first type has tensor products of two minimal models and a Liouville/cigar model. The second 
type has a single minimal model tensored with two non-compact factors. We consider supersymmetric orbifolds of these models and exhibit families of mirror pairs. The first set serves to generate an infinite set of mirror pairs (see also [10]), and illustrates in a fairly simple setting how mirror symmetry acts on elliptic genera in the non-compact case. The second set analyzes more deeply how mirror symmetry operates in the long multiplet sector. The models correspond to Gepner points in target space geometries that are close cousins to non-compact Calabi-Yau manifolds. They differ in that their asymptotics contains linear dilaton directions. The topologically non-trivial content of the geometries is coded near the origin, where we can approximate the models by an abelian orbifold of $\mathbb{C}^{3}$. The linear dilaton profile can lift some deformations of the flat model. See [10] for more details.

\subsection{The $(2 k, 2 k ; k)$ model}

From the general discussion on mirror symmetry via orbifolds, it is clear that we must identify the largest subgroup $H$ of the phase symmetries with which one can orbifold and still preserve supersymmetry. Let us perform this calculation for the $(2 k, 2 k ; k)$ model corresponding to two diagonal minimal models and one $N=2$ Liouville theory at radius $R=\sqrt{k \alpha^{\prime}}$. There exists a Landau-Ginzburg (LG) model which flows to this conformal field theory in the infrared and it is sometimes convenient to think in terms of such a description. The LG model contains three chiral superfields $X_{1}, X_{2}$ and $Y_{3}$ with superpotential:

$$
W=X_{1}^{2 k}+X_{2}^{2 k}+e^{-k Y_{3}} .
$$

The phase symmetries of the model are given by $G_{\text {phase }}=\mathbb{Z}_{2 k} \times \mathbb{Z}_{2 k} \times \mathbb{Z}_{k}$. We can identify the elements of the group $G_{\text {phase }}$ with charge vectors in the following manner. A group element corresponds to a charge vector $\gamma$ if it multiplies a state with diagonal charge vector $r$ by the phase $e^{2 \pi i \gamma \cdot r}$. We can choose generators $\gamma_{i}$ in each factor of the group $G_{p h a s e}$ such that $\gamma_{i}$ is the charge vector with entry 2 in the spot corresponding to the relevant $\mathrm{U}(1)$ charge (see definition (3.3)).

We identify the group $G=\left(\mathbb{Z}_{2 k} \times \mathbb{Z}_{2 k} \times \mathbb{Z}_{k}\right) / \mathbb{Z}_{2 k}$ as the subgroup by which we can divide after taking into account the integer R-charge orbifold $\mathbb{Z}_{n}=\mathbb{Z}_{2 k}$. The maximal subgroup $H$ of $G$ that preserves supersymmetry corresponds to charge vectors $\beta_{m}$ which are integer linear combinations of the charge vectors $\gamma_{i}$. The generators $\beta_{m}$ need to satisfy:

$$
\beta_{m}=\sum_{i} c_{m}^{i} \gamma_{i} \text { and } \beta_{m} \cdot \beta_{0} \in \mathbb{Z}
$$

Using our conventions for $\beta_{0}$, this is equivalent to

$$
\sum_{i=1}^{p} \frac{c_{m}^{i}}{k_{i}}-\sum_{j=1}^{q} \frac{c_{m}^{j}}{l_{j}} \in \mathbb{Z},
$$

where the $k_{i}$ are the levels of the minimal models while the $l_{i}$ refer to the levels of the noncompact models. The $c_{m}^{i}$ are integers. In our specific example, we have three coefficients $c_{m}^{i=1,2,3}$ for each generator $\beta_{m}$, which have to satisfy:

$$
+\frac{c_{m}^{1}}{2 k}+\frac{c_{m}^{2}}{2 k}-\frac{c_{m}^{3}}{k} \in \mathbb{Z}
$$


The integers $c_{m}^{i}$ are defined modulo $(2 k, 2 k, k)$. The integer R-charge orbifold corresponds to $c_{m}^{i}=(1,1,1)$. Thus, we can use the gauging of the integer R-charge orbifold to put the last entry to zero. Note also that if we consider the element of the R-charge orbifold group that squares to one we find that it corresponds to the vector $c_{m}^{i}=(+k,-k, 0)$. Thus, we conclude that the elements of the group $H$ have representatives where the first two entries are opposite and that these entries are only non-trivial modulo $k$. We therefore find that the group $H$ is the $\mathbb{Z}_{k}$ group generated by multiplication of the phases of $X_{1}$ and $X_{2}$ by $e^{\frac{2 \pi i}{2 k}}$ and $e^{-\frac{2 \pi i}{2 k}}$ respectively.

\subsubsection{An infinite family of mirror pairs}

To generate an infinite family of mirror pairs, we can consider subgroups of the group $H$. We suppose that the level $k$ of our initial models is equal to the product of two positive integers $k=k_{1} k_{2}$. We can then consider orbifolds of our diagonal model with the subgroup $\mathbb{Z}_{2 k} \times \mathbb{Z}_{k_{1}}$ (or strictly speaking, their semi-direct product) where the first factor corresponds to the integer R-charge orbifold and the second factor to the subgroup $\mathbb{Z}_{k_{1}}$ of the group $H$ generated by the phase multiplication $e^{ \pm 2 \pi i \frac{k_{2}}{2 k}}$ acting on the fields $X_{1,2}$. Each group element of the orbifold group is labeled by a pair of integers $(m, n)$, taking values in $\mathbb{Z}_{k}$ and $\mathbb{Z}_{k_{1}}$ respectively.

\section{Details of the calculation}

In what follows, we begin with the elliptic genus of this doubly orbifolded model and show, analogous to what was done for the $c=6$ case, that we are able to rewrite it as the elliptic genus of the mirror model. In this case, the mirror is a $\mathbb{Z}_{2 k} \times \mathbb{Z}_{k_{2}}$ orbifold of a product conformal field theory with two minimal model factors and the cigar conformal field theory.

We start out with the holomorphic part of the orbifolded elliptic genus written in terms of the twisted blocks. It depends only on the charges of the fields under the orbifold group:

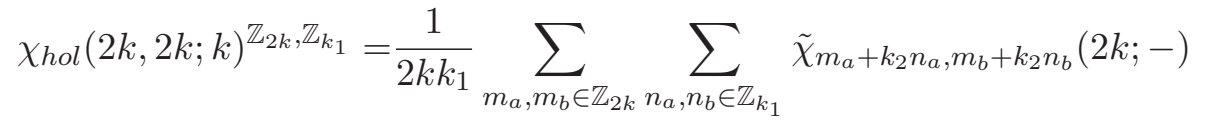

$$
\begin{aligned}
& \tilde{\chi}_{m_{a}-k_{2} n_{a}, m_{b}-k_{2} n_{b}}(2 k ;-) \tilde{\chi}_{h o l ; m_{a}, m_{b}}(-; k) .(4
\end{aligned}
$$

The first minimal model factor contributes

$$
\begin{aligned}
\tilde{\chi}_{m_{a}+k_{2} n_{a}, m_{b}+k_{2} n_{b}}(2 k ;-)= & e^{-\frac{2 \pi i\left(m_{a}+k_{2} n_{a}\right)\left(m_{b}+k_{2} n_{b}\right)}{2 k}} \\
& \times \sum_{j_{1}=0, \frac{1}{2}, \ldots}^{\frac{2 k-2}{2}} e^{\frac{2 \pi i\left(m_{b}+k_{2} n_{b}\right)\left(2 j_{1}+1\right)}{2 k}} \mathcal{C}_{2 j_{1}+1-2\left(m_{a}+k_{2} n_{a}\right)}^{j_{1}}(q, z),
\end{aligned}
$$

and similarly for the second minimal model factor with a sign flip for the $n_{a}, n_{b}$ quantum numbers. For the Liouville sector, we have

$$
\tilde{\chi}_{h o l ; m_{a}, m_{b}}(-; k)(\tau, \alpha)=e^{\frac{2 \pi i m_{a} m_{b}}{k}} \sum_{2 j_{3}-1=0}^{k-1} e^{\frac{\left(2 j_{3}-1\right)}{k} 2 \pi i m_{b}} C h\left(j_{3} ;-\frac{1}{2}+m_{a} ; q, z\right) .
$$


Putting all the factors together we obtain:

$$
\begin{aligned}
\chi_{h o l}(2 k, 2 k ; k)^{\mathbb{Z}_{2 k}, \mathbb{Z}_{k_{1}}=} & \frac{1}{2 k k_{1}} \sum_{j_{1}, j_{2}, j_{3}} \sum_{m_{a}, m_{b} \in \mathbb{Z}_{2 k}} \sum_{n_{a}, n_{b} \in \mathbb{Z}_{k_{1}}} \\
& e^{\frac{2 \pi i m_{b}}{2 k}\left(\left(2 j_{1}+1\right)+\left(2 j_{2}+1\right)+2\left(2 j_{3}-1\right)\right)} e^{\frac{2 \pi i n_{b}}{2 k_{1}}\left(\left(2 j_{1}+1\right)-\left(2 j_{2}+1\right)-2 k_{2} n_{a}\right)} \\
& \mathcal{C}_{2 j_{1}+1-2\left(m_{a}+k_{2} n_{a}\right)}^{j_{1}}(q, z) \mathcal{C}_{2 j_{2}+1-2\left(m_{a}-k_{2} n_{a}\right)}^{j_{2}}(q, z) \operatorname{Ch}\left(j_{3} ;-\frac{1}{2}+m_{a} ; q, z\right) .
\end{aligned}
$$

The sum over the twist insertion $m_{b}$ then imposes the desired integer R-charge constraint:

$$
\frac{j_{1}+j_{2}+2 j_{3}}{k} \in \mathbb{Z}
$$

A second constraint arises from the sum over the values of $n_{b}$ :

$$
\frac{j_{1}-j_{2}-k_{2} n_{a}}{k_{1}} \in \mathbb{Z}
$$

Indeed, for any projection beyond the initial integer R-charge projection, we will find a constraint between spins and a new quantum number. In order to rewrite this as the mirror elliptic genus we find it useful to eliminate the twisted quantum numbers $n_{a}$ in terms of the spin quantum numbers. In order to solve for the second constraint, recall that the angular momentum quantum number in the minimal model factor is defined modulo twice the level. Using this we find that there are precisely $k_{2}$ solutions to the second equation (where $k=k_{1} \cdot k_{2}$ ). Solving for $n_{a}$, we substitute:

$$
k_{2} n_{a}=j_{1}-j_{2}+n_{a}^{\prime} k_{1} \quad \text { with } \quad n_{a}^{\prime} \in \mathbb{Z}_{k_{2}} .
$$

This leads to

$$
\begin{aligned}
& \chi_{h o l}(2 k, 2 k ; k)^{\mathbb{Z}_{2 k}, \mathbb{Z}_{k_{1}}=} \frac{1}{2 k k_{2}} \sum_{j_{1}, j_{2}, j_{3}} \sum_{m_{a}, m_{b} \in \mathbb{Z}_{2 k}} \sum_{n_{a}^{\prime}, n_{b}^{\prime} \in \mathbb{Z}_{k_{2}}} \\
& e^{\frac{2 \pi i m_{b}}{2 k}\left(\left(2 j_{1}+1\right)+\left(2 j_{2}+1\right)+2\left(2 j_{3}-1\right)\right)} e^{\frac{2 \pi i n_{b}^{\prime}}{2 k_{2}}\left(\left(2 j_{1}+1\right)-\left(2 j_{2}+1\right)+2 k_{1} n_{a}^{\prime}\right)} \\
& \sum_{n_{a}^{\prime} \in \mathbb{Z}_{k_{2}}} \mathcal{C}_{2 j_{2}+1-2\left(m_{a}+k_{1} n_{a}^{\prime}\right)}^{j_{1}}(q, z) \mathcal{C}_{2 j_{1}+1-2\left(m_{a}-k_{1} n_{a}^{\prime}\right)}^{j_{2}}(q, z) \\
& \times C h\left(j_{3} ;-\frac{1}{2}+m_{a} ; q, z\right) .
\end{aligned}
$$

The sum over the integer $n_{b}^{\prime} \in \mathbb{Z}_{k_{2}}$ ensures that the numbers $n_{a}^{\prime}, j_{1}$ and $j_{2}$ satisfy the constraint (4.11). We now use the integer R-charge constraint to eliminate the spin $j_{2}$ in the first minimal model and the spin $j_{1}$ in the second minimal model character. After a 
shift in the $m_{a}$ variable by $-\left(2 j_{3}-1\right)$, we obtain our final expression:

$$
\begin{aligned}
\chi_{h o l}(2 k, 2 k ; k)^{\mathbb{Z}_{2 k}, \mathbb{Z}_{k_{1}}=} & \frac{1}{2 k k_{2}} \sum_{j_{1}, j_{2}, j_{3}} \sum_{m_{a}, m_{b} \in \mathbb{Z}_{2 k}} e^{-\frac{2 \pi i m_{b}}{2 k}\left[\left(2 j_{1}+1\right)+\left(2 j_{2}+1\right)+2\left(2 j_{3}-1\right)\right]} \\
& \sum_{n_{a}^{\prime}, n_{b}^{\prime} \in \mathbb{Z}_{k_{2}}} e^{\frac{2 \pi i n_{b}^{\prime}}{2 k_{2}}\left(\left(2 j_{1}+1\right)-\left(2 j_{2}+1\right)+2 k_{1} n_{a}^{\prime}\right)} \mathcal{C}_{-2 j_{1}-1-2\left(m_{a}+k_{1} n_{a}^{\prime}\right)}^{j_{1}}(q, z) \\
& \mathcal{C}_{-2 j_{2}-1-2\left(m_{a}-k_{1} n_{a}^{\prime}\right)}^{j_{2}}(q, z) C h\left(j_{3} ;-\frac{1}{2}+m_{a}-2 j_{3}-1 ; q, z\right) .
\end{aligned}
$$

Rewriting this back in terms of the twisted blocks, we find that the final expression is equal to:

$$
\begin{aligned}
\chi_{h o l}(2 k, 2 k ; k)^{\mathbb{Z}_{2 k}, \mathbb{Z}_{k_{1}}}=\frac{1}{2 k k_{2}} \sum_{m_{a}, m_{b} \in \mathbb{Z}_{2 k}} \sum_{n_{a}^{\prime}, n_{b}^{\prime} \in \mathbb{Z}_{k_{2}}} \tilde{\chi}_{m_{a}+k_{1} n_{a}^{\prime}, m_{b}+k_{1} n_{b}^{\prime}}(2 k ;-)^{\mathbb{Z}_{2 k}} \\
\tilde{\chi}_{m_{a}-k_{1} n_{a}^{\prime}, m_{b}-k_{1} n_{b}^{\prime}}(2 k ;-)^{\mathbb{Z}_{2 k}} \tilde{\chi}_{h o l ; m_{a}, m_{b}}(-; k)^{\mathbb{Z}}
\end{aligned}
$$

To infer the mirror we have more work to do. Firstly we have to ensure that the nonholomorphic part of the orbifold elliptic genus can also be written such that it is the appropriate modular completion of the above mock modular form. Secondly we need to have the orbifold of a diagonal model in order to read of the mirror.

\subsubsection{The long multiplet sector}

In order to complete our matching of elliptic genera of the mirror pairs, we also need to check the equality for the states in the long multiplet sector. For simplicity, we restrict to the case in which the levels satisfy $k_{1}=k$ and $k_{2}=1$. The generalization to the other cases is straightforward. The remainder term of the orbifold elliptic genus takes the form

$$
\begin{aligned}
\chi_{r e m}(2 k, 2 k ; k)^{\mathbb{Z}_{2 k}, \mathbb{Z}_{k}}=\frac{1}{2 k^{2}} \sum_{m_{a}, m_{b} \in \mathbb{Z}_{2 k}} \sum_{n_{a}, n_{b} \in \mathbb{Z}_{k}} \chi_{m_{a}+n_{a}, m_{b}+n_{b}}(2 k-;) \\
\chi_{m_{a}-n_{a}, m_{b}-n_{b}}(2 k-;) \chi_{r e m ; m_{a}, m_{b}}(-; k) .
\end{aligned}
$$

In order to proceed we require the twisted blocks that correspond to the non-holomorphic piece of the elliptic genus. These are given in appendix A. Using these blocks along with the expressions for the minimal model elliptic genera, we obtain

$$
\begin{aligned}
\chi_{r e m}(2 k, 2 k ; k)^{\mathbb{Z}_{2 k}, \mathbb{Z}_{k}=}=\frac{1}{2 k^{2}} \sum_{j_{1}, j_{2}} \sum_{m_{a}, m_{b} \in \mathbb{Z}_{2 k}} e^{\frac{2 \pi i m_{b}}{2 k}\left(\left(2 j_{1}+1\right)+\left(2 j_{2}+1\right)\right)} & \sum_{n_{a}, n_{b} \in \mathbb{Z}_{k}} e^{\frac{2 \pi i n_{b}}{2 k}\left(\left(2 j_{1}+1\right)-\left(2 j_{2}+1\right)-2 n_{a}\right)} \\
& \sum_{w, n \in \mathbb{Z}} e^{2 \pi i \frac{n m_{b}}{k}} z^{\frac{n-k w+2 m_{a}}{k} \mathcal{C}_{2 j_{1}+1-2\left(m_{a}+n_{a}\right)}^{j_{1}}(q, z) \mathcal{C}_{2 j_{2}+1-2\left(m_{a}-n_{a}\right)}^{j_{2}}(q, z)} \\
& (-1) \frac{1}{\pi} i \frac{\theta_{11}(\tau, \alpha)}{\eta^{3}} \int_{-\infty-i \epsilon}^{+\infty-i \epsilon} \frac{d s}{2 i s+n+k w} q^{\frac{s^{2}}{k}+\frac{\left(n-k w+2 m_{a}\right)^{2}}{4 k}} \bar{q}^{\frac{s^{2}}{k}+\frac{(n+k w)^{2}}{4 k}} .
\end{aligned}
$$


The calculation follows the same scheme as the previous one. The sum over the integers $m_{b}$ and $n_{b}$ again imposes the desired constraints:

$$
\begin{aligned}
\frac{2 j_{1}+1}{2 k}+\frac{2 j_{2}+1}{2 k}+\frac{n}{k} & \in \mathbb{Z}, \\
\frac{j_{1}}{k}-\frac{j_{2}}{k}-\frac{n_{a}}{k} & \in \mathbb{Z} .
\end{aligned}
$$

We eliminate the twisted quantum numbers $n_{a}$ in terms of the spins and obtain:

$$
\begin{aligned}
\chi_{r e m}(2 k, 2 k ; k)^{\mathbb{Z}_{2 k}, \mathbb{Z}_{k}=}= & \frac{1}{2 k} \sum_{j_{1}, j_{2}} \sum_{m_{a}, m_{b} \in \mathbb{Z}_{2 k}} e^{\frac{2 \pi i m_{b}}{2 k}\left(\left(2 j_{1}+1\right)+\left(2 j_{2}+1\right)\right)} \\
& \sum_{w, n \in \mathbb{Z}} e^{2 \pi i \frac{n m_{b}}{k} z^{\frac{n-k w+2 m_{a}}{k}} \mathcal{C}_{2 j_{2}+1-2 m_{a}}^{j_{1}}(q, z) \mathcal{C}_{2 j_{1}+1-2 m_{a}}^{j_{2}}(q, z)} \\
& (-1) \frac{1}{\pi} i \frac{\theta_{11}(\tau, \alpha)}{\eta^{3}} \int_{-\infty-i \epsilon}^{+\infty-i \epsilon} \frac{d s}{2 i s+n+k w} q^{\frac{s^{2}}{k}+\frac{\left(n-k w+2 m_{a}\right)^{2}}{4 k}} \bar{q}^{\frac{s^{2}}{k}+\frac{(n+k w)^{2}}{4 k} .}
\end{aligned}
$$

We substitute the integer $\mathrm{R}$-charge constraint in the angular momentum variable of the two minimal models and shift the variable $m_{a}$ to $m_{a}-n+k w$ and find:

$$
\begin{aligned}
\chi_{r e m}(2 k, 2 k ; k)^{\mathbb{Z}_{2 k}, \mathbb{Z}_{k}=}=\frac{1}{2 k} \sum_{j_{1}, j_{2}} \sum_{m_{a}, m_{b} \in \mathbb{Z}_{2 k}} e^{\frac{2 \pi i m_{b}}{2 k}\left(\left(2 j_{1}+1\right)+\left(2 j_{2}+1\right)\right)} & \sum_{w, n \in \mathbb{Z}} e^{2 \pi i \frac{n m_{b}}{k}} z^{\frac{-n+k w+2 m_{a}}{k}} \mathcal{C}_{-2 j_{1}-1-2 m_{a}}^{j_{1}}(q, z) \mathcal{C}_{-2 j_{2}-1-2 m_{a}}^{j_{2}}(q, z) \\
& (-1) \frac{1}{\pi} i \frac{\theta_{11}(\tau, \alpha)}{\eta^{3}} \int_{-\infty-i \epsilon}^{+\infty-i \epsilon} \frac{d s}{2 i s+n+k w} q^{\frac{s^{2}}{k}+\frac{\left(-n+k w+2 m_{a}\right)^{2}}{4 k}} \bar{q}^{\frac{s^{2}}{k}+\frac{(n+k w)^{2}}{4 k}} .
\end{aligned}
$$

We then flip the sign of the variable $m_{b}$, and find that all individual factors combined indeed agree with the twisted blocks of the mirror model:

$$
\chi_{r e m}(2 k, 2 k ; k)^{\mathbb{Z}_{2 k}, \mathbb{Z}_{k}}=\frac{1}{2 k} \sum_{m_{a}, m_{b} \in \mathbb{Z}_{2 k}} \tilde{\chi}_{m_{a}, m_{b}}(2 k ;-)^{\mathbb{Z}_{2 k}} \tilde{\chi}_{m_{a}, m_{b}}(2 k ;-)^{\mathbb{Z}_{2 k}} \tilde{\chi}_{r e m ; m_{a}, m_{b}}(-; k)^{\mathbb{Z}_{k}} .
$$

This is the modular completion of the mock modular form defined in equation (4.14) for $k_{1}=k$ and $k_{2}=1$. We thus extended the proof of the equality of elliptic genera of mirror symmetric models to the long multiplet sector.

Finally, we can rewrite the formula for the mirror elliptic genus in terms of characters which are more easily read as being associated to a diagonal spectrum. We find that the mirror can be written as:

$$
\begin{aligned}
\chi(2 k, 2 k ; k)^{\mathbb{Z}_{2 k}, \mathbb{Z}_{k}}(\tau, \alpha)=\frac{1}{2 k} \sum_{m_{a}, m_{b} \in \mathbb{Z}_{2 k}} \tilde{\chi}_{m_{a}, m_{b}}(2 k ;-)(\tau,-\alpha) \\
\tilde{\chi}_{m_{a}, m_{b}}(2 k ;-)(\tau,-\alpha) \tilde{\chi}_{m_{a}, m_{b}}(-; k)^{\mathbb{Z}_{k}}(\tau,-\alpha),
\end{aligned}
$$

where we have flipped the sign of the summation variables. We have used two facts which we already encountered while discussing the self-mirror $c=6$ example. Firstly, that the antidiagonal minimal model elliptic genera are equal to their diagonal model counterpart, up to 
an overall sign change and a change in the sign of the second argument (see equation (A.6)). Secondly, that the elliptic genus of a non-compact model is equal to itself under the sign flip of the second argument (see equation (A.21)). Note how a sign flip in the left-moving angular momentum comes down to T-duality for the compact factor, which is self-dual under T-duality. For the non-compact factor, the sign flip changes its nature from Liouville theory to cigar model.

Our final expression is consistent with our expectations about the mirror model. The original model $M_{1}$ was a $(2 k, 2 k ; k)$ model with Liouville deformation at radius $\sqrt{k \alpha^{\prime}}$. The $\mathbb{Z}_{2 k} \times \mathbb{Z}_{k_{1}}$ orbifold of the model gives rise to the mirror $M_{2}$ of this model which is a $(2 k, 2 k ; k)$ model at radius $R=\sqrt{k \alpha^{\prime}}$ modded out by $\mathbb{Z}_{2 k} \times \mathbb{Z}_{k_{2}}$. If the original model $M_{1}$ is (Liouville) deformed, then the mirror $M_{2}$ is expected to be (cigar) resolved, which is indeed the case. We have thus exhibited an infinite family of models, parameterized by a pair of integers $\left(k_{1}, k_{2}\right)$ that are mirror to one another and for which the elliptic genera match.

\subsection{The $(k ; 2 k, 2 k)$ model}

We next consider the model with two non-compact factors and one minimal model. The non-holomorphic sector of this model has qualitatively different features from the models of subsection 4.1 since it involves the modular completion of a product of two mock modular forms. The Landau-Ginzburg description of the model is given by the superpotential

$$
W=X_{1}^{k}+e^{-2 k Y_{2}}+e^{-2 k Y_{3}} .
$$

We consider the orbifold by the group $\mathbb{Z}_{2 k} \times \mathbb{Z}_{k}$ generated by:

$$
\begin{aligned}
& \left(X_{1}, e^{-Y_{2}}, e^{-Y_{3}}\right) \longrightarrow\left(e^{\frac{2 \pi i}{k}} X_{1}, e^{\frac{2 \pi i}{2 k}} e^{-Y_{2}}, e^{\frac{2 \pi i}{2 k}} e^{-Y_{3}}\right) \\
& \left(X_{1}, e^{-Y_{2}}, e^{-Y_{3}}\right) \longrightarrow\left(X_{1}, e^{\frac{2 \pi i}{2 k}} e^{-Y_{2}}, e^{-\frac{2 \pi i}{2 k}} e^{-Y_{3}}\right)
\end{aligned}
$$

For simplicity we only focus on the orbifold by the full group $H=\mathbb{Z}_{k}$.

\subsubsection{The short multiplet bound states}

Using the twisted blocks in equations (2.15) and (2.17), the holomorphic part of the elliptic genus of the double orbifold takes the form:

$$
\begin{aligned}
\chi_{h o l}(k ; 2 k, 2 k)^{\mathbb{Z}_{2 k}, \mathbb{Z}_{k}=} & \frac{1}{2 k^{2}} \sum_{j_{1}, j_{2}, j_{3}} \sum_{m_{a}, m_{b} \in \mathbb{Z}_{2 k}} \sum_{n_{a}, n_{b} \in \mathbb{Z}_{k}} \\
& e^{\frac{2 \pi i m_{b}}{2 k}\left(2\left(2 j_{1}+1\right)+\left(2 j_{2}-1\right)+\left(2 j_{3}-1\right)\right)} e^{\frac{2 \pi i n_{b}}{2 k}\left(\left(2 j_{2}-1\right)-\left(2 j_{3}-1\right)+2 n_{a}\right)} \\
& \mathcal{C}_{2 j_{1}+1-2 m_{a}}^{j_{1}} C h\left(j_{2} ;-\frac{1}{2}+\left(m_{a}+n_{a}\right) ; q, z\right) C h\left(j_{3} ;-\frac{1}{2}+\left(m_{a}-n_{a}\right) ; q, z\right) .
\end{aligned}
$$

We find the constraints:

$$
\frac{2 j_{1}+j_{2}+j_{3}}{k} \in \mathbb{Z} \quad \text { and } \quad \frac{j_{2}}{k}-\frac{j_{3}}{k}+\frac{n_{a}}{k} \in \mathbb{Z} .
$$


As before we will find it useful to eliminate the twisted quantum numbers $n_{a}$ in terms of the spins, while retaining the integer R-charge constraint as it is. We then substitute the Rcharge constraint in the angular momentum variable of the two Liouville factors. Redefining the variable $m_{a}$ variable to $m_{a}^{\prime}=m_{a}-\left(2 j_{1}-1\right)$, we finally obtain the expression:

$$
\begin{aligned}
& \chi_{h o l}(k ; 2 k, 2 k)^{\mathbb{Z}_{2 k}, \mathbb{Z}_{k}}=\frac{1}{2 k} \sum_{m_{a}, m_{b} \in \mathbb{Z}_{2 k}} \sum_{j_{1}, j_{2}, j_{3}} e^{-2 \pi i m_{b} \frac{2 j_{1}+j_{2}+j_{3}}{k}} \mathcal{C}_{-2 j_{1}-1-2 m_{a}}^{j_{1}}(q, z) \\
& C h\left(j_{2} ;-\frac{1}{2}+m_{a}-2 j_{2}-1 ; q, z\right) C h\left(j_{3} ;-\frac{1}{2}+m_{a}-2 j_{3}-1 ; q, z\right) .
\end{aligned}
$$

Repackaging this in terms of the twisted blocks, we find:

$$
\chi(k ; 2 k, 2 k)^{\mathbb{Z}_{2 k}, \mathbb{Z}_{k}}=\frac{1}{2 k} \sum_{m_{a}, m_{b} \in \mathbb{Z}_{2 k}} \tilde{\chi}_{m_{a}, m_{b}}(k-;)^{\mathbb{Z}_{k}} \tilde{\chi}_{h o l ; m_{a}, m_{b}}(-; 2 k)^{\mathbb{Z}_{2 k}} \tilde{\chi}_{h o l ; m_{a}, m_{b}}(-; 2 k)^{\mathbb{Z}_{2 k}}
$$

As was done in the earlier examples we turn now to a calculation of the non-holomorphic completion of the elliptic genus in order to read off the mirror model. The non-holomorphic contribution for this model is qualitatively different in nature and throws up new and interesting points.

\subsubsection{The long multiplet scattering states}

Schematically, the fully modular elliptic genus of this orbifold model can be decomposed into a holomorphic and non-holomorphic piece as follows: ${ }^{2}$

$$
\chi=\chi^{1} \chi^{2} \chi^{3}=\chi_{\text {hol }}^{1}\left(\chi_{\text {hol }}^{2} \chi_{\text {hol }}^{3}+\left[\chi_{\text {hol }}^{2} \chi_{\text {rem }}^{3}+\chi_{\text {rem }}^{2} \chi_{\text {hol }}^{3}+\chi_{\text {rem }}^{2} \chi_{\text {rem }}^{3}\right]\right),
$$

where we have suppressed the summation indices over the twisted blocks of the orbifold. The terms in the square parenthesis are the non-holomorphic completion for the product of two mock modular forms. The mirror analysis of the first two terms in this completion parallel the discussion in the previous subsections and we do not show the details of the calculation since we obtain the expected result parallel to the one obtained in equation (4.26). The last term is of a new type, and we consider it in detail below. Denoting it by $T_{3}$, and reinstating the missing summation indices, let us use the twisted blocks for

\footnotetext{
${ }^{2}$ We elaborate on this point in section 5.
} 
the non-holomorphic sector and write it out in full glory:

$$
\begin{aligned}
T_{3}= & \frac{1}{2 k^{2}} \sum_{m_{a}, m_{b}=0}^{2 k-1} \sum_{n_{a}, n_{b}=0}^{k-1} \tilde{\chi}_{m_{a}, m_{b}}(k ;-) \tilde{\chi}_{r e m ; m_{a}+n_{a}, m_{b}+n_{b}}(-; 2 k) \tilde{\chi}_{r e m ; m_{a}-n_{a}, m_{b}-n_{b}}(-; 2 k) \\
= & \frac{1}{2 k^{2}}\left(\frac{i}{\pi} \frac{\theta_{11}}{\eta^{3}}\right)^{2} \sum_{j_{1}} \sum_{w_{1}, n_{1}} \sum_{w_{2}, n_{2}} \sum_{m_{a}, m_{b}} \sum_{n_{a}, n_{b}} e^{\frac{2 \pi i m_{b}\left(\left(2 j_{1}+1\right)-m_{a}\right)}{k}} \mathcal{C}_{2 j_{1}+1-2 m_{a}}^{j_{1}} \\
\times & e^{\frac{2 \pi i}{2 k}\left(\left(m_{b}+n_{b}\right)\left(n_{1}+\left(m_{a}+n_{a}\right)\right)+\left(m_{b}-n_{b}\right)\left(n_{2}+\left(m_{a}-n_{a}\right)\right)\right)} \times z^{\frac{n_{1}-2 k w_{1}+2\left(m_{a}+n_{a}\right)}{2 k}+\frac{n_{2}-2 k w_{2}+2\left(m_{a}-n_{a}\right)}{2 k}} \\
& \times \int \frac{d s_{1}}{2 i s_{1}+n_{1}+2 k w_{1}} q^{\frac{s_{1}^{2}}{2 k}+\frac{\left(n_{1}-2 k w_{1}+2\left(m_{a}+n_{a}\right)\right)^{2}}{8 k}} \bar{q}^{\frac{s_{1}^{2}}{2 k}+\frac{\left(n_{1}+2 k w_{1}\right)^{2}}{8 k}} \\
& \quad \times \int \frac{d s_{2}}{2 i s_{2}+n_{2}+2 k w_{2}} q^{\frac{s_{2}^{2}}{2 k}+\frac{\left(n_{2}-2 k w_{2}+2\left(m_{a}-n_{a}\right)\right)^{2}}{8 k}} \bar{s}_{\frac{2}{2 k}+\frac{\left(n_{2}+2 k w_{2}\right)^{2}}{8 k}} .
\end{aligned}
$$

The phase factors give rise to the two constraints:

$$
\begin{aligned}
& \frac{n_{1}-2 k w_{1}}{2 k}+\frac{n_{2}-2 k w_{2}}{2 k}+\frac{2 j_{1}+1}{k} \in \mathbb{Z}, \\
& \frac{n_{1}-2 k w_{1}}{2 k}-\frac{n_{2}-2 k w_{2}}{2 k}+\frac{2 n_{a}}{2 k} \in \mathbb{Z} .
\end{aligned}
$$

We have used the fact that $n_{a}$ is defined modulo $k$. As before we can solve for the variable $n_{a}$ using the second constraint:

$$
2 n_{a}=\left(n_{2}-2 k w_{1}\right)-\left(n_{1}-2 k w_{1}\right)
$$

The variable $n_{a}$ appears in two different combinations with the other variables in both of the non-compact factors. Let us label those combinations $e_{1}$ and $e_{2}$, where

$$
e_{1}=\left(n_{1}-2 k w_{1}\right)+2\left(m_{a}+n_{a}\right) \quad \text { and } \quad e_{2}=\left(n_{2}-2 k w_{2}\right)+2\left(m_{a}-n_{a}\right) .
$$

Substituting for $n_{a}$, we see that the combinations $e_{1}$ and $e_{2}$ become

$$
e_{1}=n_{2}-2 k w_{2}+2 m_{a} \quad \text { and } \quad e_{2}=n_{1}-2 k w_{1}+2 m_{a}
$$

We use the integer R-charge constraint in equation (4.29) to obtain:

$e_{1}=-\left(n_{1}-2 k w_{1}\right)+2 m_{a}-2\left(2 j_{1}+1\right) \quad$ and $\quad e_{2}=-\left(n_{2}-2 k w_{2}\right)+2 m_{a}-2\left(2 j_{1}+1\right)$. 
Shifting the variable $m_{a}$ by $\left(-2 j_{1}-1\right)$ and substituting for the combinations $e_{i}$ in the expression for $T_{3}$, we find the final form:

$$
\begin{aligned}
T_{3}=\frac{1}{2 k}( & \left.\frac{i}{\pi} \frac{\theta_{11}}{\eta^{3}}\right)^{2} \sum_{j_{1}} \sum_{w_{1}, n_{1}} \sum_{w_{2}, n_{2}} \sum_{m_{a}, m_{b}} e^{\frac{2 \pi i m_{b}\left(2\left(2 j_{1}+1\right)+n_{1}+n_{2}\right)}{2 k}} z^{\frac{\left(-n_{1}+2 k w_{1}+2 m_{a}\right)}{2 k}+\frac{\left(-n_{2}+2 k w_{2}+2 m_{a}\right)}{2 k}} \\
& \times \int \frac{d s_{1}}{2 i s_{1}+n_{1}+2 k w_{1}} q^{\frac{s_{1}^{2}}{2 k}+\frac{\left(-n_{1}+2 k w_{1}+2 m_{a}\right)^{2}}{8 k}} \bar{q}^{\frac{s_{1}^{2}}{2 k}+\frac{\left(n_{1}+2 k w_{1}\right)^{2}}{8 k}} \\
& \times \int \frac{d s_{2}}{2 i s_{2}+n_{2}+2 k w_{2}} q^{\frac{s_{2}^{2}}{2 k}+\frac{\left.\left(-n_{2}+2 k w_{2}+2 m_{a}\right)\right)^{2}}{8 k}} \bar{q}^{\frac{s_{2}^{2}}{2 k}+\frac{\left(n_{2}+2 k w_{2}\right)^{2}}{8 k}} \mathcal{C}_{-2 j_{1}-1-2 m_{a}}^{j_{1}} \\
= & \frac{1}{2 k} \sum_{m_{a}, m_{b}} \tilde{\chi}_{h o l ; m_{a}, m_{b}}(k ;-)^{\mathbb{Z}_{k}} \tilde{\chi}_{r e m ; m_{a}, m_{b}}(-; 2 k)^{\mathbb{Z}_{2 k}} \tilde{\chi}_{r e m ; m_{a}, m_{b}}(-; 2 k)^{\mathbb{Z}_{2 k}} .
\end{aligned}
$$

The factors agree with the twisted blocks of the mirror model. Indeed, one can now combine all terms in equation (4.27) and rewrite the full elliptic genus as the integer Rcharge orbifold of an anti-diagonal minimal model at level $k$, tensored with the two cigar theories at level $2 k$. Thus, all terms in the elliptic genera confirm the mirror symmetry of the models, including the long multiplet contributions. We can also rewrite this as the elliptic genus of a diagonal minimal model combined with two cigars (up to an overall minus sign, and a minus sign in the second argument of the elliptic genus). Note how our calculation again gives rise to non-trivial identities between the orbifolded product of two modular completed Appell-Lerch sums $\hat{A}$.

Finally, let us stress that our method, ultimately based on T-duality, will work for any number of products of minimal models and Liouville/cigar theories and their orbifolds.

\section{Notes on mock modular forms}

In this section, we make various remarks on mock modular forms, a field which is in full development in both mathematics (see e.g. [29, 30]) and physics (see e.g. [31-35]). We propose that the embedding of the mathematics of mock modular forms in our present conformal field theory perspective provides a fruitful point of view.

\subsection{The shadow}

As a prelude to our discussion, it will be useful to introduce the concept of a shadow. It is sometimes convenient to make explicit the dependence of the twisted partition function (which is a real Jacobi form) on the anti-holomorphic parameter $\bar{\tau}$. Once the partition function is known, this dependence can be read off from its anti-holomorphic derivative which we refer to as the shadow [29, 30]. For starters, let us explicitly compute the shadow [29] of the elliptic genus of $N=2$ Liouville theory at radius $R=\sqrt{l \alpha^{\prime}}$ directly from the partition function [11]. ${ }^{3}$ The shadow is defined (up to normalization and conjugation)

\footnotetext{
${ }^{3}$ The shadow was also obtained in this fashion by Sameer Murthy.
} 
as the anti-holomorphic derivative of the real Jacobi form $\chi(-; l)$ :

$$
\begin{aligned}
\chi_{\text {shad }}(-; l) & =\partial_{\bar{\tau}} \chi(-; l) \\
& =-\frac{1}{4 \sqrt{l \tau_{2}}} \frac{\theta_{11}(\tau, \alpha)}{\eta^{3}} \sum_{w, n \in \mathbb{Z}} z^{\frac{n-l w}{l}}(n+l w) q^{\frac{(n-l w)^{2}}{4 l}} \bar{q}^{\frac{(n+l w)^{2}}{4 l}} \\
& =-\frac{1}{2} \sqrt{\frac{l}{\tau_{2}}} \frac{\theta_{11}(\tau, \alpha)}{\eta^{3}} \sum_{m \in \mathbb{Z}_{2 l}} \Theta_{m, l}\left(q, z^{\frac{2}{l}}\right) \Theta_{m, l}^{\frac{3}{2}}(\bar{q}),
\end{aligned}
$$

where we used the definitions of the theta-functions of weight $1 / 2$ and $3 / 2$ at level $l$ :

$$
\begin{aligned}
\Theta_{m, l}(q, z) & =\sum_{p \in \mathbb{Z}} q^{l\left(p+\frac{m}{2 l}\right)^{2}} z^{l\left(p+\frac{m}{2 l}\right)} \\
\Theta_{m, l}^{\frac{3}{2}}(q) & =\sum_{p \in \mathbb{Z}}\left(p+\frac{m}{2 l}\right) q^{l\left(p+\frac{m}{2 l}\right)^{2}} .
\end{aligned}
$$

The shadow is a sum of terms which are the product of a holomorphic theta-function of weight $1 / 2$, and an anti-holomorphic theta-function of weight $3 / 2$.

\subsection{The product of mock modular forms}

Modular forms exhibit a ring structure. In particular, the product of modular forms gives rise to another modular form. For mock modular forms, the corresponding ring structure is not yet fully understood. We therefore believe that it is interesting to observe that if mock modular forms can be interpreted as the holomorphic parts of the elliptic genera of conformal field theories, then their product can be interpreted as the holomorphic part of the elliptic genus of the tensor product conformal field theory (as in equation (2.8)). Thus, the tensor product operation on conformal field theories can give rise to a natural product of mock modular forms, or to a suggestion of how to extend the definition of mock modular forms to include these products. Clearly, the completions of these products of mock modular forms will include products of mock modular forms and remainder functions, as well as the product of remainder functions. Indeed, imagine we have two real Jacobi forms $\chi^{1,2}$, which are modular completions of mock modular forms $\chi_{\text {hol }}^{1,2}$, then their product will have a remainder term of a new type:

$$
\chi^{1} \chi^{2}=\chi_{h o l}^{1} \chi_{h o l}^{2}+\left(\chi_{h o l}^{1} \chi_{r e m}^{2}+\chi_{r e m}^{1} \chi_{h o l}^{2}+\chi_{r e m}^{1} \chi_{r e m}^{2}\right) .
$$

These sums of products of holomorphic and non-holomorphic pieces give rise to generalized shadows including the product of remainder terms (consisting of properly weighted modular integrals of theta-functions) and the shadows of individual non-compact elliptic genera (for example as in equation (5.1)).

\subsection{The orbifolds of completions of mock modular forms}

We gave an explicit example of an orbifold of such a product of completed mock modular forms in subsection 4.2. It is clear that our construction gives rise to a large class of real Jacobi forms that is non-trivial. The corresponding mock modular forms may contain 
multiple poles. ${ }^{4}$ Beyond the orbifolds discussed in this paper, we can imagine many different types of mock modular forms and their completions that can arise in physical contexts. Instead of performing R-charge orbifolds as we have done up to now, we can extend the orbifold group much further.

For instance, we can consider symmetric product orbifold groups. It is straightforward to write down the elliptic genus of a symmetric product orbifold, using its Lagrangian description in terms of a sum over coverings of the torus by the torus. The result is a new Jacobi form obtained from the seed through Hecke operators. The Hamiltonian interpretation of the resulting formula could prove interesting. Moreover, we can introduce discrete torsion in more general abelian or non-abelian orbifolds, further enlarging the class of expressions that one can obtain on the physics side, providing more examples of what could be called (generalized) mock modular forms.

Yet another class of theories that can be examined, are Landau-Ginzburg theories with mixes of polynomial potentials, and exponentials. One can compute their elliptic genus using free field techniques. For the polynomials, one uses the techniques of [1] while for the exponentials, one uses the approach of [11]. This could potentially open up a whole new realm of mock modular forms, corresponding to elliptic genera of conformal field theories that may not be exactly solvable but that can be described as infrared limits of supersymmetric field theories.

\subsection{Uniqueness}

Since the mathematics of mock modular forms is not yet set in stone, it is harder at the moment to prove the uniqueness of modular completions of the largest class of mock modular forms (see however [28-30] for interesting results in this direction). In particular, the approach (used for compact models) of identifying polar parts and using ellipticity and modularity to prove equality of elliptic genera is not yet available for generic completed mock modular forms (though it may apply to the case of a single non-compact factor examined in subsection 4.1.2). Such a general mathematical theory could give rise to the physical statement that the long multiplet sector matching is guaranteed by ellipticity and modularity. That would provide interesting information on the asymptotics of these non-compact Gepner models from their bound state spectrum, and vice versa.

\section{Acknowledgments}

We would like to thank Atish Dabholkar, Jeff Harvey, Amir Kashani-Poor, Albrecht Klemm, Sameer Murthy and Thomas Wotschke for interesting discussions and useful correspondence. We thank the authors of [28] for making a preliminary version of their work available to us. S.A would like to thank the Chennai Mathematical Institute for hospitality during the completion of this work. Our research is partly funded by the grant ANR-09BLAN-0157-02.

\footnotetext{
${ }^{4}$ The typical shadow however will be different from the shadow for the double pole case discussed in [28], where it is the sum of a product of holomorphic and anti-holomorphic modular forms. We thank Sameer Murthy for a discussion on this point.
} 


\section{A Characters}

\section{A.1 Minimal model characters}

One way to define $N=2$ minimal model characters is implicitly:

$$
\sum_{n \in \mathbb{Z}_{2 k}} \mathcal{C}_{n}^{j(s)}(\tau, \alpha) \Theta_{n, k}\left(\tau,-\frac{2 \alpha}{k}\right)=\chi^{j}(\tau, 0) \Theta_{s, 2}(\tau,-\alpha) .
$$

We used the theta-functions defined by the formula:

$$
\Theta_{n, k}(\tau, \alpha)=\sum_{m \in \mathbb{Z}} e^{2 \pi i \tau k\left(m+\frac{n}{2 k}\right)^{2}} e^{2 \pi i \alpha k\left(m+\frac{n}{2 k}\right)} .
$$

The Ramond sector ground states correspond to states with R-charges $\pm((2 j+1) / k-1 / 2)$. The characters for representations built on ground states are $\mathcal{C}_{2 j+1}^{j(+1)}$ and $\mathcal{C}_{-2 j-1}^{j(-1)}$. We note that these two lists are in fact identical when we use the equivalence relation $(j, n, s) \equiv$ $(k / 2-j-1, n+k, s+2)$. From their implicit definition, we find the character transformation rule:

$$
\mathcal{C}_{n}^{j(s)}\left(\tau, \alpha+m_{a} \tau+m_{b}\right)=q^{-\frac{c}{6} m_{a}^{2}} z^{-\frac{c}{3} m_{a}} e^{2 \pi i\left(\frac{n}{k}-\frac{s}{2}\right) m_{b}} \mathcal{C}_{n-2 m_{a}}^{j\left(s-2 m_{a}\right)}(\tau, \alpha) .
$$

We also need the twisted Ramond sector characters $\mathcal{C}_{n}^{j}$ which we define as:

$$
\mathcal{C}_{n}^{j}=\mathcal{C}_{n}^{j(1)}-\mathcal{C}_{n}^{j(-1)} .
$$

They satisfy the transformation rule:

$$
\mathcal{C}_{n}^{j}\left(\tau, \alpha+m_{a} \tau+m_{b}\right)=(-1)^{m_{a}+m_{b}} q^{-\frac{c}{6} m_{a}^{2}} z^{-\frac{c}{3} m_{a}} e^{2 \pi i \frac{n}{k} m_{b}} \mathcal{C}_{n-2 m_{a}}^{j}(\tau, \alpha),
$$

as well as the equality:

$$
\mathcal{C}_{-n}^{j}(\tau, \alpha)=-\mathcal{C}_{n}^{j}(\tau,-\alpha) .
$$

\section{A.2 Minimal model twisted blocks}

In computing the minimal model twisted blocks, we assume that for an individual model we have a partition function in which we sum over left and right spins which satisfy $s=\bar{s}$ modulo 2. This is a diagonal sum in terms of NS and R sectors. We then find for the elliptic genus:

$$
\chi(k ;-)=\frac{\theta_{11}\left(q, z^{\frac{k-1}{k}}\right)}{\theta_{11}\left(q, z^{\frac{1}{k}}\right)}=\sum_{j=0, \frac{1}{2}, \ldots}^{\frac{k-2}{2}} \mathcal{C}_{2 j+1}^{j}(q, z) .
$$

The twisted blocks are:

$$
\begin{aligned}
\chi_{m_{a}, m_{b}}(k ;-) & =e^{2 \pi i \frac{c}{6} m_{a} m_{b}} e^{2 \pi i \frac{c}{6}\left(m_{a}^{2} \tau+2 m_{a} \alpha\right)} \sum_{j=0, \frac{1}{2}, \ldots}^{\frac{k-2}{2}} \mathcal{C}_{2 j+1}^{j}\left(\tau, \alpha+m_{a} \tau+m_{b}\right) \\
& =e^{2 \pi i \frac{c}{6} m_{a} m_{b}}(-1)^{m_{a}+m_{b}} \sum_{j=0, \frac{1}{2}, \ldots}^{\frac{k-2}{2}} e^{2 \pi i m_{b} \frac{2 j+1}{k}} \mathcal{C}_{2 j+1-2 m_{a}}^{j}(\tau, \alpha) .
\end{aligned}
$$


Inserting the standard phase $\epsilon$, we obtain

$$
\tilde{\chi}_{m_{a}, m_{b}}(k ;-)=e^{-\frac{2 \pi i m_{a} m_{b}}{k}} \sum_{j=0, \frac{1}{2}, \ldots}^{\frac{k-2}{2}} e^{2 \pi i m_{b} \frac{2 j+1}{k}} \mathcal{C}_{2 j+1-2 m_{a}}^{j}(\tau, \alpha) .
$$

We have used the known elliptic properties of the Ramond sector characters in order to derive the twisted blocks. Equivalently, we can perform the calculation using the ellipticity properties of the theta-function. We obtain

$$
\tilde{\chi}_{m_{a}, m_{b}}(k ;-)=z^{-\frac{m_{a}}{k}} \frac{\theta_{11}\left(z^{\left(1-\frac{1}{k}\right)} q^{-\frac{m_{a}}{k}} e^{-\frac{2 \pi i m_{b}}{k}}\right)}{\theta_{11}\left(z^{\frac{1}{k}} q^{\frac{m_{a}}{k}} e^{\frac{2 \pi i m_{b}}{k}}\right)} .
$$

We note in passing that with this choice of phase $\epsilon$, the twisted blocks of [14] and [16] agree. It remains to compare this to the sum of the Ramond sector characters. We rewrite:

$$
\tilde{\chi}_{m_{a}, m_{b}}(k ;-)=z^{-\frac{m_{a}}{k}} \frac{\theta_{11}\left(z^{\prime\left(1-\frac{1}{k}\right)} q^{-m_{a}} ; q\right)}{\theta_{11}\left(z^{\prime \frac{1}{k}} ; q\right)},
$$

with

$$
z^{\prime}=z q^{m_{a}} e^{2 \pi i m_{b}}
$$

Using the elliptic property of the theta-function, we can write this as

$$
\tilde{\chi}_{m_{a}, m_{b}}(k ;-)=(-1)^{m_{a}} q^{\frac{m_{a}^{2}}{2}\left(1-\frac{2}{k}\right)} z^{m_{a}\left(1-\frac{2}{k}\right)} e^{-\frac{2 \pi i m_{a} m_{b}}{k}} \frac{\theta_{11}\left(z^{\left(1-\frac{1}{k}\right)} ; q\right)}{\theta_{11}\left(z^{\prime \frac{1}{k}} ; q\right)} .
$$

The ratio of theta functions can be expanded in terms of the Ramond-sector characters as in equation (A.7). We then again use the elliptic properties of the minimal model characters (A.5) to find that the result agrees with equation (A.9). We have come full circle.

\section{A.3 The $\mathbb{Z}_{k}$ orbifold and mirror symmetry}

Consider the $\mathbb{Z}_{k}$ orbifold the $N=2$ minimal model (with $s=\bar{s} \bmod 2$ ) of central charge $c=3-6 / k$. Let us calculate the elliptic genus of the orbifold:

$$
\begin{aligned}
\chi(k ;-)^{\mathbb{Z}_{k}} & =\frac{1}{k} \sum_{m_{a}, m_{b} \in \mathbb{Z}_{k}} \tilde{\chi}_{m_{a}, m_{b}}(k ;-) \\
& =\frac{1}{k} \sum_{m, n \in \mathbb{Z}_{k}} e^{-2 \pi i \frac{m_{a} m_{b}}{k}} \sum_{j=0, \frac{1}{2}, \ldots}^{\frac{k-2}{2}} e^{2 \pi i m_{b} \frac{2 j+1}{k}} \mathcal{C}_{2 j+1-2 m_{a}}^{j}(\tau, \alpha) .
\end{aligned}
$$

The sum over the variable $m_{b}$ puts $m_{a}=2 j+1(\bmod k)$ and adds a factor of $k$. We can most easily eliminate $m_{a}$ from the sum and find:

$$
\begin{aligned}
\chi(k ;-)^{\mathbb{Z}_{k}} & =\sum_{j=0, \frac{1}{2}, \ldots}^{\frac{k-2}{2}} \mathcal{C}_{-2 j-1}^{j}(\tau, \alpha) \\
& =-\sum_{j=0, \frac{1}{2}, \ldots}^{\frac{k-2}{2}} \mathcal{C}_{2 j+1}^{j}(\tau,-\alpha) .
\end{aligned}
$$


This is one of the simplest examples of mirror symmetry in conformal field theory. We recognize the previous to last line as the elliptic genus of the anti-diagonal minimal model. Note that for these compact models, the $\mathbb{Z}_{k}$ orbifold is equivalent to performing T-duality.

\section{A.4 Characters at $c>3$}

The elliptic genus of $N=2$ Liouville theory at radius $R=\sqrt{l \alpha^{\prime}}$ contains a holomorphic part and a remainder term, namely it is a completed Appell-Lerch sum $\hat{A}_{2 l}$ :

$$
\begin{aligned}
\chi(; l) & =\chi_{h o l}+\chi_{r e m} \\
& =i \frac{\theta_{11}(\tau, \alpha)}{\eta^{3}} \hat{A}_{2 l}\left(z^{\frac{1}{l}}, z^{2} ; q\right) \\
\chi_{h o l}(; l) & =i \frac{\theta_{11}(\tau, \alpha)}{\eta^{3}} \sum_{m \in \mathbb{Z}} \frac{q^{l m^{2}} z^{2 m}}{1-z^{\frac{1}{l}} q^{m}} \\
\chi_{r e m}(; l) & =-\frac{1}{\pi} i \frac{\theta_{11}(\tau, \alpha)}{\eta^{3}} \sum_{w, n \in \mathbb{Z}} z^{\frac{n-l w}{l}} \int_{-\infty-i \epsilon}^{+\infty-i \epsilon} \frac{d s}{2 i s+n+l w} q^{\frac{s^{2}}{l}+\frac{(n-l w)^{2}}{4 l}} \bar{q}^{\frac{s^{2}}{l}+\frac{(n+l w)^{2}}{4 l}(\mathrm{~A} .16)}
\end{aligned}
$$

The holomorphic part of the Liouville elliptic genus can be expanded in terms of the twisted Ramond sector characters. We have the equation:

$$
\begin{aligned}
\chi_{h o l}(-; l) & =\frac{i \theta_{11}(q, z)}{\eta^{3}} \sum_{m \in \mathbb{Z}} \frac{q^{l m^{2}} z^{2 m}}{1-z q^{l m}} \sum_{2 j-1=0}^{l-1} z^{\frac{2 j-1}{l}} q^{m(2 j-1)} \\
& =\sum_{2 j-1=0}^{l-1} C h\left(j ;-\frac{1}{2} ; q, z\right) .
\end{aligned}
$$

We notice that the elliptic genus is expressed as a sum over extended characters. These correspond to ordinary characters summed over spectral flow orbits that shift the angular momentum quantum number by multiples of the level $l$.

The holomorphic part of the cigar elliptic genus can also be written in terms of these extended characters:

$$
\begin{aligned}
\chi_{h o l}(-; l)^{\mathbb{Z}_{k}}(q, z) & =\sum_{m=0,1, \ldots l-1} \sum_{w} \frac{i \theta_{11}(q, z)}{\eta^{3}} \frac{q^{l w^{2}-m w} z^{2 w-\frac{m}{l}}}{1-z q^{l w-m}} . \\
& =\sum_{2 j-1=0}^{k-1} C h\left(j ;-\frac{1}{2}-(2 j-1) ; q, z\right) .
\end{aligned}
$$

The modular and ellipticity properties of the extended characters are (for $m_{a}, m_{b} \in \mathbb{Z}$ ):

$$
C h\left(j ; r^{\prime} ; q, z q^{m_{a}} e^{2 \pi i m_{b}}\right)=(-1)^{m_{a}+m_{b}} q^{-\frac{c}{6} m_{a}^{2}} z^{-\frac{c}{3} m_{a}} e^{\frac{2 \pi i m_{b}\left(2 j+2 r^{\prime}\right)}{l}} C h\left(j ; r^{\prime}+m_{a} ; q, z\right) .
$$

The angular momentum of the representations corresponding to these characters is $2 j+2 r^{\prime}$. We also have the following transformation rules for the holomorphic and remainder term 
of the elliptic genus:

$$
\begin{aligned}
\chi_{h o l}(-; l)(\tau,-\alpha) & =\chi_{h o l}(-; l)(\tau, \alpha)-\frac{i \theta_{11}}{\eta^{3}} \sum_{m \in \mathbb{Z}} q^{k m^{2}} z^{2 m} \\
\chi_{r e m}(-; l)(\tau,-\alpha) & =\chi_{r e m}(-; l)(\tau, \alpha)+\frac{i \theta_{11}}{\eta^{3}} \sum_{m \in \mathbb{Z}} q^{k m^{2}} z^{2 m} .
\end{aligned}
$$

The extra term is a reminder of the ambiguity of the holomorphic part of the elliptic genus, due to the bound state spectrum touching the delta-function normalizable continuum. Together, these equations give rise to the equality:

$$
\chi(; l)(\tau,-\alpha)=\chi(; l)(\tau, \alpha),
$$

which can also be derived directly from the integral representation of the non-compact elliptic genus.

\section{A.5 Twisted building blocks at $c>3$}

\section{A.5.1 Character formulae}

Using these properties, we can calculate the holomorphic part of the twisted blocks for the Liouville and cigar elliptic genera:

$$
\begin{aligned}
\chi_{m_{a}, m_{b}}(-; l)(\tau, \alpha) & =e^{2 \pi i \frac{c}{6} m_{a} m_{b}} e^{2 \pi i \frac{c}{6}\left(m_{a}^{2} \tau+2 m_{a} \alpha\right)} \sum_{2 j-1=0}^{l-1} C h\left(j ;-\frac{1}{2} ; \tau, \alpha+m_{a} \tau+m_{b}\right) \\
& =(-1)^{m_{a}+m_{b}} e^{2 \pi i \frac{c}{6} m_{a} m_{b}} \sum_{2 j-1=0}^{l-1} e^{2 \pi i m_{b} \frac{2 j-1}{l}} C h\left(j ;-\frac{1}{2}+m_{a} ; \tau, \alpha\right) .
\end{aligned}
$$

We use the value of the central charge $c=3+6 / l$, multiply by the phase factor $\epsilon$ and obtain:

$$
\tilde{\chi}_{h o l ; m_{a}, m_{b}}(-; l)=e^{\frac{2 \pi i m_{a} m_{b}}{l}} \sum_{2 j-1=0}^{l-1} e^{\frac{(2 j-1)}{k} 2 \pi i m_{b}} C h\left(j ;-\frac{1}{2}+m_{a} ; q, z\right) .
$$

For the cigar, we find:

$$
\tilde{\chi}_{h o l ; m_{a} \cdot m_{b}}(-; l)^{\mathbb{Z}_{l}}=e^{\frac{2 \pi i m_{a} m_{b}}{l}} \sum_{2 j-1=0}^{l-1} e^{\frac{-(2 j-1)}{l} 2 \pi i m_{b}} C h\left(j ;-\frac{1}{2}-(2 j-1)+m_{a} ; q, z\right) .
$$

\section{A.5.2 Twisted blocks for the non-holomorphic sector}

For the continuous character part of the elliptic genus we find, for the Liouville theory twisted block:

$$
\begin{aligned}
\tilde{\chi}_{r e m ; m_{a}, m_{b}}(-; l)=(-1) \frac{1}{\pi} i \frac{\theta_{11}(\tau, \alpha)}{\eta^{3}} \sum_{w, n \in \mathbb{Z}} e^{2 \pi i \frac{\left(n+m_{a}\right) m_{b}}{l}} z^{\frac{n-l w+2 m_{a}}{l}} \\
\int_{-\infty-i \epsilon}^{+\infty-i \epsilon} \frac{d s}{2 i s+n+l w} q^{\frac{s^{2}}{l}+\frac{\left(n-l w+2 m_{a}\right)^{2}}{4 l}} \bar{q}^{\frac{s^{2}}{l}+\frac{(n+l w)^{2}}{4 l}} .
\end{aligned}
$$


For the cigar theory, we end up with:

$$
\begin{aligned}
\tilde{\chi}_{r e m ; m_{a}, m_{b}}(-; l)^{\mathbb{Z}_{l}}=e^{2 \pi i \frac{m_{a} m_{b}}{l}}(-1) & \frac{1}{\pi} i \frac{\theta_{11}(\tau, \alpha)}{\eta^{3}} \sum_{w, n \in \mathbb{Z}} e^{-2 \pi i m_{b} \frac{n}{l}} z^{-\frac{n-l w}{l}+\frac{2 m_{a}}{l}} \\
& \int_{-\infty-i \epsilon}^{+\infty-i \epsilon} \frac{d s}{2 i s+n+l w} q^{\frac{s^{2}}{l}+\frac{\left(n-l w-2 m_{a}\right)^{2}}{4 l}} \bar{q}^{\frac{s^{2}}{l}+\frac{(n+l w)^{2}}{4 l} .} .
\end{aligned}
$$

\section{A.5.3 Exact expressions for twisted blocks}

Finally, we give the expressions for the complete twisted building blocks, for the Liouville theory:

$$
\tilde{\chi}_{m_{a}, m_{b}}(-; l)=e^{\frac{2 \pi i m_{a} m_{b}}{l}} q^{\frac{m_{a}^{2}}{l}} z^{\frac{2 m_{a}}{l}} \frac{i \theta_{11}(\tau, \alpha)}{\eta^{3}} \hat{A}_{2 l}\left(z^{\frac{1}{l}} q^{\frac{m_{a}}{l}} e^{\frac{2 \pi i m_{b}}{l}}, z^{2} q^{2 m_{a}} ; q\right),
$$

and for the cigar theory at radius $R=\sqrt{l \alpha^{\prime}}$ :

$$
\begin{aligned}
\tilde{\chi}_{m_{a}, m_{b}}(-; l)^{\mathbb{Z}_{l}}= & e^{\frac{2 \pi i m_{a} m_{b}}{l}} q^{\frac{m_{a}^{2}}{l}} z^{\frac{2 m_{a}}{l}} \frac{i \theta_{11}(\tau, \alpha)}{\eta^{3}} \\
& \times \frac{1}{l} \sum_{m_{a}^{\prime}, m_{b}^{\prime} \in \mathbb{Z}_{l}} q^{-\frac{m_{a}^{\prime 2}}{l}} e^{-\frac{2 \pi i m_{a}^{\prime} n_{a}^{\prime}}{l}} \hat{A}_{2 l}\left(z^{\frac{1}{l}} q^{\frac{m_{a}+m_{a}^{\prime}}{l}} e^{\frac{2 \pi i\left(m_{b}+m_{b}^{\prime}\right)}{l}}, z^{2} q^{2 m_{a}} ; q\right) .
\end{aligned}
$$

Open Access. This article is distributed under the terms of the Creative Commons Attribution License which permits any use, distribution and reproduction in any medium, provided the original author(s) and source are credited.

\section{References}

[1] E. Witten, On the Landau-Ginzburg description of $N=2$ minimal models, Int. J. Mod. Phys. A 9 (1994) 4783 [hep-th/9304026] [INSPIRE].

[2] E. Witten, Phases of $N=2$ theories in two-dimensions, Nucl. Phys. B 403 (1993) 159 [hep-th/9301042] [INSPIRE].

[3] K. Hori et al., Mirror symmetry, Clay Mathematics Monographs.

[4] O. Aharony, M. Berkooz, D. Kutasov and N. Seiberg, Linear dilatons, NS five-branes and holography, JHEP 10 (1998) 004 [hep-th/9808149] [INSPIRE].

[5] A. Giveon, D. Kutasov and O. Pelc, Holography for noncritical superstrings, JHEP 10 (1999) 035 [hep-th/9907178] [INSPIRE].

[6] T. Eguchi and Y. Sugawara, Modular invariance in superstring on Calabi-Yau n fold with ADE singularity, Nucl. Phys. B 577 (2000) 3 [hep-th/0002100] [INSPIRE].

[7] T. Eguchi and Y. Sugawara, D-branes in singular Calabi-Yau $n$ fold and $N=2$ Liouville theory, Nucl. Phys. B 598 (2001) 467 [hep-th/0011148] [INSPIRE].

[8] T. Eguchi and Y. Sugawara, $S L(2, \mathbb{R}) / U(1)$ supercoset and elliptic genera of noncompact Calabi-Yau manifolds, JHEP 05 (2004) 014 [hep-th/0403193] [INSPIRE].

[9] T. Eguchi and Y. Sugawara, Conifold type singularities, $N=2$ Liouville and $S L(2: R) / U(1)$ theories, JHEP 01 (2005) 027 [hep-th/0411041] [INSPIRE]. 
[10] S.K. Ashok, R. Benichou and J. Troost, Non-compact Gepner Models, Landau-Ginzburg Orbifolds and Mirror Symmetry, JHEP 01 (2008) 050 [arXiv:0710.1990] [INSPIRE].

[11] J. Troost, The non-compact elliptic genus: mock or modular, JHEP 06 (2010) 104 [arXiv: 1004.3649] [INSPIRE].

[12] T. Eguchi and Y. Sugawara, Non-holomorphic Modular Forms and $S L(2, \mathbb{R}) / U(1)$ Superconformal Field Theory, JHEP 03 (2011) 107 [arXiv: 1012.5721] [INSPIRE].

[13] S.K. Ashok and J. Troost, A Twisted Non-compact Elliptic Genus, JHEP 03 (2011) 067 [arXiv:1101.1059] [INSPIRE].

[14] T. Kawai, Y. Yamada and S.-K. Yang, Elliptic genera and $N=2$ superconformal field theory, Nucl. Phys. B 414 (1994) 191 [hep-th/9306096] [INSPIRE].

[15] P. Di Francesco, O. Aharony and S. Yankielowicz, Elliptic genera and the Landau-Ginzburg approach to $N=2$ orbifolds, Nucl. Phys. B 411 (1994) 584 [hep-th/9306157] [INSPIRE].

[16] P. Berglund and M. Henningson, Landau-Ginzburg orbifolds, mirror symmetry and the elliptic genus, Nucl. Phys. B 433 (1995) 311 [hep-th/9401029] [INSPIRE].

[17] P. Berglund and S.H. Katz, Mirror symmetry constructions: A review, hep-th/9406008 [INSPIRE].

[18] T. Kawai and S.-K. Yang, Duality of orbifoldized elliptic genera, Prog. Theor. Phys. Suppl. 118 (1995) 277 [hep-th/9408121] [INSPIRE].

[19] A. Schellekens and N. Warner, Anomalies and modular invariance in string theory, Phys. Lett. B 177 (1986) 317 [INSPIRE].

[20] E. Witten, Elliptic genera and quantum field theory, Commun. Math. Phys. 109 (1987) 525 [INSPIRE].

[21] P. Di Francesco and S. Yankielowicz, Ramond sector characters and $N=2$ Landau-Ginzburg models, Nucl. Phys. B 409 (1993) 186 [hep-th/9305037] [INSPIRE].

[22] K. Hori and A. Kapustin, Duality of the fermionic $2-D$ black hole and $N=2$ Liouville theory as mirror symmetry, JHEP 08 (2001) 045 [hep-th/0104202] [INSPIRE].

[23] D. Israel, A. Pakman and J. Troost, D-branes in $N=2$ Liouville theory and its mirror, Nucl. Phys. B 710 (2005) 529 [hep-th/0405259] [INSPIRE].

[24] S.K. Ashok, S. Murthy and J. Troost, D-branes in non-critical superstrings and minimal super Yang-Mills in various dimensions, Nucl. Phys. B 749 (2006) 172 [hep-th/0504079] [INSPIRE].

[25] D. Gepner, Space-Time Supersymmetry in Compactified String Theory and Superconformal Models, Nucl. Phys. B 296 (1988) 757 [InSPIRE].

[26] B.R. Greene and M. Plesser, Duality in Calabi-Yau moduli space, Nucl. Phys. B 338 (1990) 15 [inSPIRE].

[27] D. Israel, C. Kounnas, A. Pakman and J. Troost, The Partition function of the supersymmetric two-dimensional black hole and little string theory, JHEP 06 (2004) 033 [hep-th/0403237] [INSPIRE].

[28] A. Dabholkar, S. Murthy and D. Zagier, unpublished.

[29] S. Zwegers, Mock Theta functions, PhD thesis, Utrecht University, The Neatherlands (2002). 
[30] D. Zagier, Ramanujan's mock theta functions and their applications d'après Zwegers and Bringmann-Ono, Séminaire Bourbaki (2007) 986.

[31] A. Semikhatov, A. Taormina and I.Y. Tipunin, Higher level Appell functions, modular transformations and characters, math/0311314 [INSPIRE].

[32] D. Gaiotto, G.W. Moore and A. Neitzke, Four-dimensional wall-crossing via three-dimensional field theory, Commun. Math. Phys. 299 (2010) 163 [arXiv:0807.4723] [INSPIRE].

[33] M. Alim et al., Wall-crossing holomorphic anomaly and mock modularity of multiple M5-branes, arXiv:1012.1608 [INSPIRE].

[34] J. Manschot, BPS invariants of $N=4$ gauge theory on a surface, arXiv:1103.0012 [INSPIRE].

[35] M.-x. Huang, A.-K. Kashani-Poor and A. Klemm, The Omega deformed B-model for rigid $N=2$ theories, arXiv:1109.5728 [INSPIRE]. 